Revue européenne des sciences sociales

European Journal of Social Sciences

XXXIX-120 | 2001

Autour de l'épistémologie de la sociologie et de la sociologie des sciences: des débats, un réexamen

\title{
Intorno alle discussioni e ricerche recenti sulla sociologia delle scienze
}

\section{Giovanni Busino}

\author{
(2) OpenEdition \\ Journals \\ Edizione digitale \\ URL: http://journals.openedition.org/ress/661 \\ DOI: $10.4000 /$ ress.661 \\ ISSN: $1663-4446$ \\ Editore \\ Librairie Droz

\section{Edizione cartacea} \\ Data di pubblicazione: 1 luglio 2001 \\ Paginazione: 145-189 \\ ISBN: 2-600-00610-9 \\ ISSN: 0048-8046
}

Notizia bibliografica digitale

Giovanni Busino, "Intorno alle discussioni e ricerche recenti sulla sociologia delle scienze », Revue européenne des sciences sociales [En ligne], XXXIX-120 | 2001, mis en ligne le 14 décembre 2009, consulté le 19 avril 2019. URL : http://journals.openedition.org/ress/661 ; DOI : 10.4000/ress.661 


\section{Giovanni BUSINO}

\section{INTORNO ALLE DISCUSSIONI E RICERCHE RECENTI SULLA SOCIOLOGIA DELLE SCIENZE}

\section{INTRODUZIONE}

Gli studi sulla scienza, e sulle diverse discipline che la compongono, sono stati finora riservati alle cure dei filosofi, degli storici e dei sociologi. Le relazioni e gli scambi tra queste tre categorie di studiosi sono stati purtroppo sempre rari ed in generale infruttuosi, o comunque poco profittevoli. Le rispettive tradizioni e preoccupazioni, fortemente distinte, hanno condizionato le problematiche della filosofia, della storia e della sociologia delle scienze e fatto sì che queste tre discipline si sviluppassero autarchicamente, senza tener conto dei risultati ottenuti in settori di ricerca più o meno contigui.

Mentre la filosofia ha costantemente tentato di rendere espliciti i fondamenti della scienza (concezione della natura, successione dei sistemi metafisici ed epistemologici) in quanto sistema coerente di conoscenze oggettive, d'elaborare delle classificazioni delle diverse pratiche scientifiche, di caratterizzarne le specificità, le condizioni d'esistenza e di validità, rispetto a tutte le altre forme di conoscenze e di saperi umani, di rivelarne i processi genetici e di sviluppo ${ }^{1},-$ la sociologia s'è attribuita il compito di analizzare la metascienza ed i rapporti dei saperi scientifici con i fattori esistenziali, culturali, sociali, ambientali, di descrivere le strutture organizzative e professionali, i modelli di distribuzione sociale del potere, la produzione dei significati culturali, indispensabili all'esercizio della ricerca. Perciò la sociologia, influenzata soprattutto dai lavori d'orientamento weberiano di Robert K. Merton, ha lasciato ad altre discipline lo studio delle caratteristiche interne della scienza (coerenza logica, conferma empirica, osservazione sperimentale, interazione delle teorie della scienza con altri sistemi di pensiero) e si è riservato quello delle caratteristiche esterne, del funzionamento delle istituzioni scientifiche e delle loro proprietà (l'universalismo, il comunalismo, il disinteresse, lo scetticismo organizzato). Per i sociologi della corrente mertoniana, corrente detta anche «classica » 0 «moderata », la scienza è universale perché i suoi metodi sono impersonali ed oggettivi, perché ricerca la verità guidata dalla logica e dall'osser-

Granger, G.-G., La science et les sciences, Paris, PUF, 1993. Recentemente è stato proposto d'assegnare alla filosofia della scienza lo studio delle verità ed alla sociologia della conoscenza scientifica i presupposti erronei delle credenze scientifiche.Ved. a questo proposito Fillieule, R., Un modèle d'analyse des présupposés en sociologie de la connaissance scientifique, in «Revue européenne des sciences sociales », XXXVI, 1998, n 111, pp. 199-215 nonché Lecuyer, B.-P. Sociologie de la connaissance et sociologie de la science, in: Sous la direction de Kremer-Marietti A., Sociologie de la science et rationalité scientifique, Sprimont, Mardaga, 1998, pp. 17-45. 
vazione, settori di ricerca, quest'ultimi, riservati però al lavoro di ricostruzione razionale dei filosofi ${ }^{2}$. La storia, invece, non ha mai definito nettamente, almeno sino a pochi anni or sono, il settore di sua competenza. Infatti, il lavoro dello storico, s'è concentrato innanzitutto sul locale ed il singolare, sulla vita, sulle opere degli scienziati, sulla peculiarità dei diversi campi della ricerca scientifica, sulla diversità dei metodi scientifici, ed ha mirato a ricostruire le mentalità, le idee e la cultura di un'epoca e d'un luogo, l'evoluzione delle singole discipline ed il loro ruolo, considerato come il prodotto di forze politiche e d'interessi personali, nei processi di sviluppo della civiltà. Se le ricerche sulle funzioni d'istituzioni quali le Società, le Accademie, i Laboratori, le Scuole, ecc., sulle funzioni dell'insegnamento e della formazione alla ricerca, sulle applicazioni pratiche e sulle implicazioni sociali delle scienze nella vita quotidiana, sono numerose, al contrario sono sporadiche quelle sui metodi per l'ottenimento dell'oggettività, della validità, per distinguire gli interessi sociali dalle verità scientifiche, il razionale dall'irrazionale, insomma tutto ciò che caratterizza e particolarizza i contenuti cognitivi delle scienze. Rarissime sono, inoltre, le analisi storiografiche rivolte a stabilire la natura delle correlazioni tra le scienze formali, le scienze sperimentali e la tecnologia nonché i loro rispettivi tratti caratteristici ${ }^{3}$. È noto che la storia delle tecniche si è sviluppata finora nell'indifferenza o nell'ignoranza di quella delle scienze !

Tale tradizionale tripartizione è stata fortemente contestata, sin dagli inizi degli anni '60, da taluni filosofi ${ }^{4}$ e da numerosi sociologi ${ }^{5}$. Per entrambi la scienza

2 Per una rapida presentazione della tematica ved. Busino, G., Sociologie des sciences et des techniques, Paris, PUF, 1998. Per un'esposizione più scolastica: Vinck, D., Sociologie des sciences, Paris, Colin, 1995, ed un'accurata storia della disciplina, delle teorie e dei dibattiti il documentato lavoro di Dubois, M., Introduction à la sociologie des sciences et des connaissances scientifiques, Paris, PUF, 1999. Ved. inoltre i testi fondatori di Merton, R. K., Social Theory and Social Structure, New York, Free Press, 1957 e The Sociology of Science. Theoretical and Empirical Investigations, Chicago, University of Chicago Press, 1973 e il saggio di Plé, B., La sociologie de la science de Merton: une prise de conscience américaine à l'égard du progrès de la science, in: Sous la direction de Kremer-Mariotti, A., Sociologie de la science, op. cit., pp. 93-114.

3 Agassi, J., Towards an Historiography of Science, in «History and Theory. Studies in the Philosophy of History », vol. 2, n. 3, 's-Gravenhage, Mouton, 1963; Russo, F., Nature et méthode de l' histoire des sciences, Paris, Blanchard, 1983 e dello stesso i preziosi Eléments de bibliographie de l'histoire des sciences et des techniques, Paris, Hermann, 1969, 2a ed. Vedere altresi Moscovici, S., L' histoire des sciences et la science des historiens, in «Archives européennes de sociologie», VII, 1966, pp. 116-126, nonché Roger, J., Pour une histoire des sciences à part entière. Introduction de C. Blanckaert, postface de Jean Gayon, Paris, Albin Michel, 1995; Sous la direction de Guesnerie, R. e Hartog, F., Des Sciences et des Techniques: un débat, in «Cahiers des Annales », n 45, Paris. Ed.de l’EHESS, 1998, spec. le sezioni I, III e IV.

4 Per una buona rassegna critica ved. Chalmers A., What is this Thing Called Science? An Assessment of the Nature and Status of Science and its Methods, St. Lucia, University of Queensland Press, 1976; second edition, 1982; Id., Science and its Fabrication, Buckingham, Open University Press, 1990, nonché la raccolta Elements d'histoire des sciences. Sous la direction de M. Serres, Paris, Bordas, 1989; nuova ed., Paris, Larousse-Bordas, 1997. Cfr. il «manifesto» di Latour, B., Philosophie contre sociologie des sciences. Une querelle enfin dépassée?, «Le débat», n 92, novembre-décembre 1996, pp. 153-163 e l'arringa «pro domo sua »: Les chantiers actuels des études sociologiques sur les sciences exactes, in: Sous la direction de Guesnerie, R. e Hartog, F., Des Sciences et des Techniques : un débat, op. cit., pp. 11-24.

5 Per un rapido panorama, cfr. Busino, G., La sociologie de la science de R. K. Merton à D. Bloor et B. Latour, Lausanne, IASUL, 1994; Id., Questions actuelles de sociologie de la science, Lausanne, IASUL, 1995; Id., La sociologie de la connaissance scientifique, Lausanne, IASUL, 1997; Id., Sociologie des sciences et des techniques, op. cit. 
non è né autarchica né autonoma, ma piuttosto un fatto sociale totale. Un'aspra critica s'è sviluppata anche tra quegli storici desiderosi d'analizzare le maniere secondo cui le scienze vengono architettate, attenti a delucidare le procedure sperimentali d'attribuzione e stabilizzazione dei significati degli enunciati, a ricostruire le modalità per pervenire a risolvere le controversie ed imporre una soluzione, a ricostituire gli sviluppi e le implicazioni techniche, politiche e sociali delle attività scientifiche e a rivelare, infine, i rapporti tra la politica, l'economia e la ricerca scientifica ${ }^{6}$.

Alle origini di questi cambiamenti e delle problematiche derivatene, peraltro ambedue ben descritti ed analizzati da diversi autori ${ }^{7}$, si trovano innanzitutto i lavori di Thomas S. Khun (1922-1996) e di quegli studiosi che ne hanno ripreso e poi sviluppato, da angolazioni particolari e secondo prospettive differenti, le tematiche, ed anche, seppure in maniera indiretta, $\mathrm{i}$ «cultural studies stories ${ }^{8}$.

Si sa che gli studi culturali sono una critica spietata del «cultural Engineering», della cultura funzionale alle esigenze della regolazione e del controllo sociali. Dalle loro ricostruzioni critiche gli studiosi fanno discendere la necessità di abbattere le frontiere tuttora esistenti tra le diverse discipline umanistico-sociali e quelle scientifiche ed anche il dovere di mettere a disposizione dei cittadini le conoscenze e gli strumenti indispensabili per comprendere il mondo, per contestarlo ed eventualmente per cambiarlo.

6 Per un accurato excursus storico cfr. Castelli Gattinara, E., Epistémologie, Histoire et Histoire des sciences dans les années 1930, in «Revue de synthèse », n 1, janviers-mars 1998, pp. 9-61; Id., Les inquitétudes de la raison. Epistémologie et histoire en France entre les deux guerres, Paris, EHESS/Vrin, 1998, e per i dibattiti contemporanei l'ottima rassegna di Pestre, D., Pour une histoire sociale et culturelle des sciences. Nouvelles définitions, nouveaux objets, nouvelles pratiques, in «Annales HSS», mai-juin 1995, n³, pp. 487-522 e dello stesso Les «Social Studies of Science» et leurs effets sur le travail historique, in «Raison présente», n 119, pp. 35-46; Les sciences et l' histoire aujourd' hui, «Le débat», n 102, pp. 53-68; Sciences des philosophes et sciences des historiens, Ibid., pp. 99-106. Dello stesso tre esempi di ricerca concreta: Louis Néel, le magnétisme et Grenoble, Paris, Ed. du CNRS, 1990; Physique et physiciens en France, 19181940, Paris, Editions des archives contemporaines, 1992, IIa ed., et Les physiciens dans les sociétés occidentales de l'après-guerre. Une mutation des pratiques techniques et des comportements sociaux et culturels, in «Revue d'histoire moderne et contemporaine», janviers-mars $1992, \mathrm{n}^{\circ} 1$, pp. 56-72. Cfr. altresì Dalmedico, A. D. \& Pestre, D., Comment parler des sciences aujourd' hui?, in: Sous la direction de Jourdant, B., Impostures scientifiques. Les malentendus de l'affaire Sokal, Paris, La Découverte/Alliage, 1998, pp. 77-105.

7 Per esempio, tra tanti altri, in maniera incisiva, da Boudon, R., La nuova sociologia della conoscenza scientifica, nella sua raccolta Il vero e il giusto. Saggi sull'obiettività dei valori e della conoscenza, Bologna, Il Mulino, 1997, pp. 283-317, nonché da Bunge, M., A critical Examination of the New Sociology of Science, in «Philosophy of the Social Science», XXI, 1991, n 4, pp. 524560 e XXII, 1992, n 1 , pp. 46-76, e poi in maniera più sistematica nel libro Social Science under Debate: A philosophical Perspective, Toronto, University of Toronto Press, 1998. Ved. altresi Chaudury, M., La rationalité scientifique, un réexamen, in: Sous la direction de Kremer-Mariotti, A., Sociologie de la science, op. cit., pp. 171-194 nonché il magistrale e fondamentale saggio di Hacking, I., The Social Construction of what?, Cambridge, MA, Harvard University Press, 1999.

8 Brantlinger, P., Crusoe's Footprint. Cultural Studies in Britain and America, London, Routledge, 1990; Chaney, D., The culural Turn, London, Routledge, 1994; Davies, I., Cultural Studies and Bayond. Fragments of Empire, London, Routledge, 1995. Per una rassegna bibliografica in materia ved. Mattelart, A. \& Neveu, E., «Cultural studies' Stories». La domestication d' une pensée sauvage?, in «Réseaux », 1996, n 80, pp. 11-58. 
Tale radicalismo teorico e politico attribuisce agli studi culturali la dignità di scienza regina, la sola scienza atta a rendere compiutamente conto dei meccanismi sociali e delle strutture socioculturali, concepiti come un tutto, come delle totalità fortemente integrate.

I lavori di Kuhn, duramente criticati da Karl R. Popper, da John Walkins, ma anche da Paul F. Feyerabend, da Imre Lakatos e da tanti altri, sono recepiti, riletti, re-interpretati da filosofi, storici e sociologi, tutti affascinati dalla tesi della storicità della scienza e da quella del convenzionalismo dei linguaggi scientifici. La critica della nozione di progresso scientifico, la sua sostituzione con quella di crescita della scienza, l'introduzione della distinzione «scienza normale / scienza eccezionale» (extraordinary science), dei concetti di «paradigma», di «rivoluzione scientifica», sono ritenuti apporti considerevoli per ben formare una nuova immagine della scienza, per spiegarne e svelarne il funzionamento ed anche per legittimare le rivendicazioni di democratizzazione della vita sociale.

L'attività scientifica non è caratterizzata dalle discussioni critiche degli enunciati e dalla verifica logico-sperimentale delle proposizioni, bensì dal fatto che un insieme di postulati, di concetti, di teorie, di metodi, di convenzioni, di credenze, di dogmi, accomunano i ricercatori d'uno stesso settore e li integrano in una comunità solidale. Quivi le competenze danno autorità e prestigio, determinano la legittimità dei problemi esemplari, delle soluzioni tipiche e delle applicazioni riuscite in un settore determinato della realtà. Quando cogli strumenti intellettuali a disposizione non si riesce più a ridurre, ad assorbire, a neutralizzare le anomalie apparse nel corso d'una ricerca, allora la contestazione del paradigma risulta inevitabile. Allo scopo di risolvere i problemi insoluti, si ricostruisce e si ristruttura bruscamente (Gestalt switch) l'intero campo del sapere con altri principi, metodi e techniche. I nuovi approcci attraggono gruppi di ricercatori, che li fanno propri e così facendo danno lo spunto alla nascita d'un nuovo paradigma. Scelto ed accettato per ragioni estranee alla logica e alla sperimentazione, il nuovo paradigma è una soluzione, per così dire, irrazionale del problema del cambiamento d'una autorità razionale. Può anche non risolvere le anomalie che hanno fatto abbandonare il vecchio paradigma. In contrasto coll'impegno conoscitivo interiorizzato nel corso dei processi di formazione e di socializzazione professionali e mediante i quali avviene l'integrazione e la consolidazione delle comunità scientifiche, gli scienziati che optano per il nuovo paradigma agiscono in maniera nonlogica, sulla base di motivazioni extra-scientifiche, di una reazione emotiva.

La transizione da un paradigma ad un altro paradigma resta un avvenimento brutale, una sorta di rivoluzione nella scienza, un mutamento della visione del mondo, una trasformazione della Gestalt. Una tale transizione introduce una discontinuità radicale nella storia della scienza.

I paradigmi, mutevoli nel tempo, nella maniera di risolvere i problemi, d'organizzare i rapporti degli uomini col mondo esterno, sono perciò incomparabili e incommensurabili. Per questa ragione i saperi scientifici non sono cumulativi né costituiscono un progresso suscettibile d'attestare l'oggettività del mondo reale'

9 Urry, J., Thomas S. Kuhn as sociologist of Knowledge, in «British Journal of Sociology », XXIV, 1973, n4, pp.462-473. Per la specifica ricezione delle opere kuhniane tra i culturi di scienze umane importante è il libro di Barnes, B., T.S.Kuhn and Social Science, New York, Columbia University Press, 1982, nonché Callon, M. e Latour, B., Introduction, in La science telle qu' elle se fait. Anthologie de la sociologie des sciences de langue anglaise, Paris, La Découverte, 1991, pp. 7-36. 
Poco tempo prima di morire, Kuhn, sconcertato da queste interpretazioni, dichiarò pubblicamente di rifiutare il relativismo, la riduzione delle sue dottrine a pura sociologia della conoscenza, di non accettare il dissolvimento dei concetti di verità e di ragione, la subordinazione della ricerca a considerazioni politiche, la confusione tra i saperi scientifici ed i saperi socio-culturali. E rivendicò anche, con molta energia, il diritto per la filosofia di riflettere sulla specificità della conoscenza e della razionalità, sulla nozione di significato e sulla natura e le ragioni della sua variabilità nel tempo e nello spazio ${ }^{10}$.

Questa netta presa di posizione non ha avuto alcun effetto sui sociologi e storici lettori delle opere di Kuhn ${ }^{11}$, i quali continuano a rifiutare la distinzione tra $\mathrm{i}$ fattori sociali, ideologici, e gli aspetti scientifici; tutti persistono nel rifiuto del metodo scientifico universale, della riproducibilità naturale delle verifiche per mezzo degli esperimenti, e difendono con accanimento la tesi che la storia sociale delle scienze debba soprattutto ricostruire la procedure mediante le quali le teorie sono «costruite», «fabbricate», nei laboratori, e poi fatte valere all'interno delle comunità di scienziati ${ }^{12}$.

Per tutti questi studiosi la finalità degli studi sociali delle scienze è di scoprire in che maniera le convenzioni e le procedure sono state elaborate, come sono state valutate le conoscenze e poi come sono state dotate di significatività. Gli aspetti teorici, materiali, tecnici, strumentali del lavoro scientifico sono così immersi nel sociale e confusi col politico ${ }^{13}$. In questo modo, la natura della conoscenza, i suoi modi di costituzione e di riproduzione, i suoi legami coi contesti sociali e storici, diventano i principali temi di ricerca.

Non più sistema di verità costantemente in progresso, non più ortogenesi della ragione, la scienza è, per molti di questi studiosi, un sapere con pretese teleologiche, un sapere non molto dissimile da tutti gli altri saperi; è l'ideologia della cultura moderna dell'Occidente capitalista. Presunta impropriamente universale, vera, oggettiva, autonoma, normativa, valida per tutta l'Umanità, la scienza, è così ridotta a sapere costruito in un momento ed in una situazione particolari, è dipendente da strumenti specifici, è importante a causa delle sue pratiche concrete e non già per i discorsi teorici e giustificativi degli scienziati, a causa delle sue dimensioni performative e non già per i suoi contenuti cognitivi, per il contesto sociale e storico in cui i fatti scientifici sono stati costruiti e non già per i principi assoluti

10 Delacampagne, Ch., Un entretien avec Thomas S. Kuhn. «La vérité scientifique n'a pas besoin d'être unique», «Le Monde», 5-6 février 1995, p.13. Cfr. anche Tschannen, O., Paradigms and Exemplars in Sociology: a Kuhnian Reformulation, «Revue suisse de sociologie», 20, 1994, $\mathrm{n}^{\circ} 2$, pp. 463-486; Ascher, E., Piaget, Kuhn et l'herméneutique double, «Revue européenne des sciences sociales », XXV, 1987, n 74, pp. 61-90.

11 A questo proposito si rimanda a Matalon, B., Sociologie de la science et relativisme, in « Revue de synthèse», IVe s., $n^{\circ} 3$, juillet-septembre 1986, pp. 267-290 e dello stesso La construction de la science. De l'épistémologie à la sociologie de la connaissance scientifique, Lausanne, Delachaux \& Niestlé, 1996.

12 Per una rassegna dei dibattiti e delle problematiche in materia, ved. la miscellanea diretta da Pickering, A., Science as Practice and Culture, Chicago, Chicago University Press, 1992.

13 Tra i tanti lavori rappresentativi di questo indirizzo, citiamo, a titolo di esempio, Latour, B., Les microbes. Guerre et Paix, suivi de Irréductions, Paris, Métailié, 1984, e Licoppe, Ch., La formation de la pratique scientifique. Le discours de l' expérience en France et en Angleterre (16301820), Paris, La Découverte, 1996. 
le cui radici vanno ricercate nella metascienza e nella metafisica occidentali, nella loro forza egemonica ${ }^{14}$.

\section{IL «PROGRAMMA FORTE»}

Nel 1974 esce il libro di Barry Barnes Scientific Knowledge and Scientific Theory, un vero e proprio manifesto contro l'egemonia della sociologia di Merton, della pretesa del suo «programma debole» che la spiegazione sociologica riguarda qualsiasi forma di deviazione, e contro la filosofia analitica per la quale solo l'errore deve essere spiegato mentre la verità deriva automaticamente dalla logica.

Pochi anni prima una «Science Studies Unit» è creata, nell'ambito dell'Università di Edimburgo, da David Bloor e da Barry Barnes, che la dotano anche d'un periodico, subito divenuto famoso, le «Social Studies of Science ${ }^{15}$.

Influenzati dai lavori di Kuhn, interpretati però alla luce della fenomenologia, dello strutturalismo, del postmodernismo e della filosofia del secondo Wittgestein, quello del Tractatus Logico-Philosophicus (1921), i ricercatori della «SSU» criticano crudamente la filosofia di Popper e degli empiristi logici, proclamano che non esistono basi empiriche per giustificare la validità della conoscenza scientifica, contestano la distinzione neo-positivista tra la logica della scoperta e la logica della giustificazione, rifiutano le dicotomie forma/fondo, interno/esterno, e fanno valere la tesi che la scienza è una pratica inseparabile dal culturale, dal sociale e dal politico da cui è stata ed è ognora istituita ${ }^{16}$. Essa serve

14 Cfr. Woolgar, S., On the alleged Distinction between Discourse and Praxis, «Social Studies of Science», 16, 1986, pp. 309-317. Una discussione critica dei temi accennati nel testo si trova in Simon, G., De la reconstitution du passé. A propos de l' histoire des sciences, entre autres histoires, «Le débat», $\mathrm{n}^{\circ}$ 66, septembre-octobre 1991, pp. 134-147 e De l' histoire des sciences aux savoirs contemporains. Entretien avec Gérard Simon, «Le débat», $\mathrm{n}^{\circ} 101$, novembre-décembre 1998, pp. 107-130. Per un'ottima discussione delle teorie dell'evidenza e della giustificazione e della dicotomia internalismo/esternalismo, si rimanda a Haack, S., Evidence and Inquiry. Toward Reconstruction in Epistemology, Oxford, Blackwell, 1993. Sull'universalizzazione dei particolarismi propri ad una tradizione storica singolare e sui meccanismi della circolazione internazionale delle idee, ved. Bourdieu, P. e Wacquant, L., Sur les ruses de la raison impérialiste, «Actes de la recherche en sciences sociales », $n^{\circ} 121-122$, mars 1998, pp. 109-118.

15 I lavori di Barnes e Bloor si trovano elencati nella bibliografia posta in appendice a Bloor, D., Knowledge and Social Imagery, Chicago, University of Chicago Press, 1991, e poi anche nel manuale di Barnes, B., Bloor, D. \& Henry, John, Scientific Knowledge. A Sociological Analysis, London, Athlone, 1996, pp. 215-225. Un'eccellente discussione delle tesi difese in questo manuale si trova in Mermin, N. D., The science of science. A physicist Reads Barnes, Bloor and Henry, «Social Studies of Science», 28, 1998, n 4, pp. 603-623. Bloor è l'autore di due libri sul filosofo e logico austriaco di nazionalità inglese: Wittgenstein. A Social Theory of Knowledge, London, Macmillan, 1983 e Wittgenstein, Rule and Institutions, London, Routledge, 1997. Recentemente egli ha scritto un'apologia del «programma forte», della sua vicinanza alle dottrine di Sir F. C. Barlett e della tesi che qualsiasi enunciato debba render conto della logica della sua credibilità in Remember the «Strong Programme»? Le "programme fort» à l'épreuve de la mémoire, in «Enquête», n 5, Premier semestre 1997, pp. 55-68.

16 Collins, H. M., The place of the «core-set» in modern Science: social Contingency with methodological Propriety in Science, «History of Science», 19, 1981, pp. 6-19; Boisvert, Y., L'analyse post-moderniste: une nouvelle grille d'analyse socio-politique, Montréal, Harmattan Inc., 1997. 
a giustificare ed a legittimare il controllo sociale; essa è determinata e plasmata dai saperi della società in cui opera. La componente sociale è sempre presente e costitutiva della conoscenza, ma ciò non vuol dire che ci siano relazioni sistematiche tra una teoria e la sua collocazione sociale. Le teorie atomistiche della fisica non sono il prodotto necessario della società individualista.

Bloor sostiene che bisogna «indagare e spiegare il contenuto e la natura della conoscenza scientifica», senza mai distinguerla «dagli eventi che ne determinano la produzione». «Non esistono limitazioni insite nella natura assoluta o trascendente della conoscenza scientifica in quanto tale, o nella natura specifica della razionalità, della validità, della verità o dell'oggettività ${ }^{17}$. Ogni forma di conoscenza, comprese le parti più astratte, è sempre sociale.

Questa sorta di convenzionalismo e di strumentalismo fa presumere che tutte le culture siano uguali, che tutti i sistemi verbali possiedano delle basi razionali, che non esistano né forme né gradi diversi di conoscenza, che l'adozione d'una teoria, o d'una dottrina, o d'una classificazione, sia una scelta utilitaria, una risposta più o meno efficace ad una contingenza, ad una congiuntura, ad una pratica $^{18}$. Bisogna perciò demistificare la scienza e le sue pretese d'universalità, d'autonomia e di neutralità; bisogna insistere sul fatto che esiste una pluralità di scienze, che i metodi, le procedure ed i risultati delle inchieste scientifiche sono dei prodotti culturalmente e storicamente marcati, che le questioni finora trattate dagli epistemologi sono insignificanti giacché mirano essenzialmente a sacralizzare la natura e la portata d'un solo approccio della realtà, quello scientifico. Tutti i sistemi cognitivi correnti in una società, hanno un'identica legittimità. La scienza, il mito, la religione, la magia non hanno altre fondamenta se non quelle che la società loro attribuisce. Perciò essi sono equivalenti. E precisamente per questa ragione, la ricerca sulla scienza deve basarsi su quattro caposaldi: (a) la causalità, che impone di ricostruire le condizioni di produzione dei diversi sistemi di credenze partendo dalle loro cause sociali. Queste ultime possono essere molteplici e coesistere purché l'explicans non faccia riferimento alla validità della credenza esaminata. La spiegazione deve necessariamente far ricorso al determinismo; (b) l'imparzialità per la quale le credenze vere e le credenze false, razionali e irrazionali, con o senza successo pragmatico, debbono essere analizzate nella stessa maniera ove siano ammesse e riconosciute da un gruppo sociale; (c) la simmetria impone che le credenze vere e le credenze false, logiche e non logiche, efficaci ed inefficaci, debbano essere spiegate unicamente sulla base della medesima causa: (d) la riflessività ingiunge d'applicare lo stesso schema esplicativo alla scienza ed agli studi sociali delle scienze.

La spiegazione causale, l'imparzialità, la simmetria e la riflessività sarebbero le condizioni sine qua non dell'approccio naturalista per lo studio della conoscenza in generale e della scienza in particolare. Esse solo spiegherebbero perché crediamo nelle verità della scienza, cioè in proprietà semantiche indipendenti dal

17 Basti qui citare di Bloor, D., Knowledge and Social Imagery, op.cit., la cui prima edizione uscì nel 1976. Le dottrine di Bloor sono discusse ed analizzate da vari autori in una raccolta a cura di Brown, J.R., Scientific Rationality. The sociological Turn, Dordrecht, Reidel, 1984.

18 Barnes, B., Scientific Knowledge and Scientific Theory, London, Routledge \& Kegan Paul, 1974; Interests and the Growth of Knowledge, London, Routledge \& Kegan Paul, 1977. 
fatto di credere in queste verità. Dato che possiamo credere in idee vere per ragioni non vere ed in idee non vere per ragioni vere e valide, non si può non concludere che le proprietà semantiche non bastano a fondare il perché crediamo nelle verità della scienza.

Per Bloor non esiste una conoscenza scientifica diversa da quella ordinaria, quella del senso comune e della vita quotidiana. Distinguere ed opporre le due forme di conoscenza è un pregiudizio impostoci dai modelli di spiegazione deduttivi. La conoscenza può essere garantita dall'analogia e dall'estensione metaforica di tutti i modelli concettuali esistenti e fondati socialmente.

Se qualsiasi conoscenza è la risultante di cause sociali, bisogna ritenere queste ultime sempre vere e incontestabili, o soltanto efficaci?

Il principio di simmetria della spiegazione causale elimina questo interrogativo, che concerne la verità o la falsità delle credenze spiegate. Per Bloor, affermare che una credenza è vera o falsa non ha niente a che vedere col fatto che essa sia o non determinata da una causa sociale. La spiegazione causale resta valida in sé e per sé, indipendentemente dai fattori ai quali s'applica, e la conoscenza è sempre quella che gli attori sociali definiscono tale in un contesto sociale dato.

La scienza non può auto-fondarsi colle sue proprie procedure, non può controllare la validità delle sue produzioni né autodescriversi in maniera coerente, senza incappare in un circolo vizioso. I suoi criteri non permettono, infatti, di distinguere la scienza dalla non scienza. Derivati da valori particolari, questi criteri sono equivalenti, per quanto riguarda i fondamenti della loro validità, dagli altri universi cognitivi fondati su valori differenti. Per queste ragioni, il concetto di razionalità è variabile. La scelta d'una tra le teorie rivali non è mai neutra. Le teorie fanno parte d'un contesto. Quando il contesto cambia, il significato dei termini d'una teoria cambia ugualmente. Il confronto tra i termini di due teorie è impossibile. Ognuno di questi termini ha una portata ed un peso diversi a causa delle differenze esistenti tra i contesti.

Barnes e Bloor affermano che l'obiettivo razionalista di produrre elementi di conoscenza con un'affidabilità universale e nello stesso tempo giustificabili senza far ricorso al contesto, questo obiettivo è irrealizzabile ${ }^{19}$. Essi però non dicono cosa siano la natura ed il contenuto delle conoscenze né perché quest'ultime siano esclusivamente delle costruzioni sociali. Inoltre, l'applicazione meccanica del modello causale, la sua unicità, non permette di distinguere la natura ed il contenuto sociali dalle procedure utilizzate per la spiegazione, lo schema logico dalle proprietà di questo costruttivismo ontologico, di dirci se trattisi di determinazione causale, d'influenza esteriore, di condizionamento o di pura e semplice corrispondenza.

Bloor si limita a ripetere che solo la descrizione delle operazioni di produzione ci consente di conoscere come e perché pensiamo ed agiamo, come e perché i nostri pensieri acquisiscono, conservano o perdono lo statuto di conoscenza. In

19 Barnes, B. \& Bloor, D., Relativism, Rationalism and the Sociology of Knowledge, in Hollis, M. \& Lukes, S. Ed., Rationality and Relativism, Oxford, Blackwell, 1982, pp. 21-47 e Turnbull, D., Relativism, Reflexivity and the Sociology of Scientific Knowledge, «Metascience», 1984, n 1-2, pp. 47-60. Un'ottima analisi critica dei dibattiti cui hanno dato luogo queste controversie si trova in Laudan, L., Science and Relativism. Some Key Controversies an the Philosophy of Science, Chicago, University of Chicago Press, 1990. 
conseguenza, le idee relative alla conoscenza sono delle rappresentazioni sociali, la necessità logica è una forma d'obbligo morale e l'oggettività un fenomeno sociale. Le fondamenta della scienza sono allora delle credenze, congetturali e teoriche, prodotte dalle procedure per l'elaborazione del consenso e per il regolamento delle controversie, procedure a loro volta generate dalle strutture sociali e politiche ${ }^{20}$. Scrive Bloor: «Nulla è assoluto e definitivo». «Perciò ogni conoscenza è relativa alla situazione locale dei pensatori che la producono. Le idee e le congetture che sono in grado di produrre, i problemi che li assillano, il gioco reciproco di assunti e critiche nel loro ambiente, i loro scopi e i loro obiettivi, le loro esperienze, i criteri e i significati che adottano: che cosa sono tutti questi fattori se non fattori determinanti naturalistici di credenze [...]?»

L'osservazione dipende dai nostri presupposti per cui i risultati non sono neutri. Non c'è una soluzione algoritmica alle dispute scientifiche. Per ogni osservazione esiste un numero infinito di spiegazioni potenziali e non disponiamo d'una teoria per confermare le proposizioni così formulate.

Si può dire che questo problema dei fondamenti o presupposti essenziali, del consenso a proposito della scelta d'una teoria, sia così difficile ed esiziale che il fondatore del «programma forte» ce lo tratteggia?

Il teorema di Kurt Gödel e la storia delle scienze, ricca di teoremi non dimostrati che continuano ad essere studiati, come, per esempio, l'ultimo teorema di Fermat, dovrebbe indurci ad essere più cauti e ad ammettere, in prima approssimazione, che la logica della dimostrazione è assai diversa da quella dell'argomentazione. Gli scienziati non si prefiggono come scopo assoluto di costruire dei sistemi cognitivi incondizionatamente validi e capaci di autofondarsi completamente, ma unicamente di rispettare il principio di non-contraddizione e di costruire dei sistemi validi condizionatamente alla validità di altri sistemi.

Quando si dice che la validità è il proprio di tutti i sistemi di credenze, che è una funzione del sistema sociale, allora siamo privati della possibilità di controllare empiricamente la dipendenza dal sistema sociale dei sistemi di credenze. In più, se si riduce la validità d'una proposizione alla sua credibilità sociale, non c'è più modo d'accertare ciò che avviene nel contesto della validazione/giustificazione e ciò che è attribuito dal contesto sociale d'origine e dall'accettazione hic et nunc. Ci troviamo nell'impossibilità d'analizzare le regole e le pratiche che consentono di parlare della verità/validità/razionalità di questa o quella proposizione ed inoltre di chiarire in che maniera avviene l'attribuzione della credibilità alle regole ed alle decisioni susseguenti che ne derivano, d'esplorarne la sintassi e la semantica.

Per i partigiani del «programma forte» non c'è alcuna relazione tra l'esperienza, la logica ed il linguaggio, non esiste una razionalità specifica, interna alla

\footnotetext{
Una radicalizzazione di queste tesi è stata effettuata da Collins, H., Stages in the empirical Program of Relativism, «Social Studies of Science», 11, 1981, pp. 3-11. Gli studi storici disponibili non permettono però di ritenerla plausibile. Per esempio, una ricerca storica recente sul contrasto e le rivalità tra due Scuole di medicina, tra il 1817 ed il 1852, mostra che gli interessi professionali, le opzioni filosofiche e politiche, il differenziale della produttività scientifica incidono sui contenuti scientifici più di qualsiasi determinismo sociale. Ved. a questo proposito Raynaud, D., La controverse entre organicisme et vitalisme. Etude de sociologie des sciences, «Revue française de sociologie », XXXIV, 1998, n 4, pp. 721-750.
} 
scienza ed al suo sviluppo. Tutto è dato dalla visione del mondo del gruppo sociale d'appartenenza, dalle sue razionalizzazioni ideologiche. La scienza è simile alla religione. Il «potere della forza collettiva», delle «potenze esteriori », « dei principi che organizzano la società » le attribuisce autorità, forza, influenza e prestigio $^{21}$.

Il paradosso che si cela dietro queste assunzioni, è considerevole. I quattro principi che fondano il «programma forte» (spiegazione causale di tutte le credenze, imparzialità rispetto alla verità ed alla falsità, simmetria per la spiegazione causale di tutte le conoscenze, riflessività dei modelli di spiegazione) presuppongono l'esistenza d'una natura che precede la scienza e la religione e impongono di trattare queste in maniera equivalente. Se però si dice che la scienza è l'espressione delle strutture sociali, economiche e politiche, allora non possiamo più fondarla sulla natura ed i quattro principi sono inapplicabili. Se si dice che la scienza è l'esteriorizzazione delle strutture sociali e dei loro principi organizzativi, allora la detta scienza non è se non l'espressione della società. Nell'un caso e nell'altro sia la natura che la società sono reificate ed il naturalismo dichiarato è un «trompe-l'oeil».

Bloor scrive: «Il nostro pensiero, nel suo insieme, suppone istintivamente che noi esistiamo in un ambiente esteriore comune che possiede una struttura determinata». «La verità, la razionalità e la validità» sono dei fini naturali degli uomini ed orientano le loro tendenze naturali. Gli uomini ragionerebbero «naturalmente in maniera giusta e accetterebbero la verità logica allorché la constaterebbero ». Siccome non esiste una struttura sociale senza una struttura neuronale, gli uomini hanno delle inclinazioni naturali all'induzione ed alla deduzione.

Queste asserzioni hanno un senso se postuliamo l'esistenza d'una natura umana dotata d'una logica che permetta di sceverare la razionalità oggettiva (corrispondenza al reale) dalla razionalità formale(consistenza logica), natura umana fornita di certe facoltà, d'un certo numero di capacità d'adattamento, d'assimilazione, d'accomodamento, dell'istinto delle combinazioni. Ma se affermiamo che tutta l'esperienza in quanto azione, costruzione e strutturazione progressive di ciò che ci circonda, è il prodotto d'influenze e di fattori sociali, la logica naturale e le facoltà che ne derivano non sono più degli elementi costitutivi della natura umana bensì il prodotto d'una costruzione sociale. In questo caso, non soltanto non si può accedere individualmente ed autonomamente ad una razionalità indipendente dalle costrizioni sociali ma bisogna anche risolvere o chiarire il mistero della natura di quest'essere collettivo preesistente ai singoli individui e dotato di capacità logiche e di facoltà antecedenti a tutti i processi di strutturazione sociale ${ }^{22}$.

21 Il riferimento alla dottrina di Emile Durkheim è esplicito. Ved. Bloor, D., Durkheim and Mauss Revisited. Classification and the Sociology of Knowledge, «Studies in History and Philosophy of Science», 13, 1982, pp.267-297, nonché Némedi, D., Durkheim and the «Strong Programme» in the Philosophy of Science, «Revue européenne des sciences sociales», XXVIII, 1990, n 88, pp. 55-75.

22 Lara, Ph. de, Un mirage sociologique. La «construction sociale de la réalité», «Le débat », $\mathrm{n}^{\circ} 97$, novembre-décembre 1997, pp. 114-129; Corcuff, Ph., Entre malentendus sociologiques et impensé politique. Réponse à Philippe de Lara, Ibid., n 103, janvier-février 1999, pp. 112-120; Lara, Ph. de, Nouvelle sociologie ou vieille philosophie?, Ibid., pp. 121-129. Un'analisi critica pertinente delle dottrine delle mentalità collettive si trova in Lloyd, G. E. R., Demystifying mentalities, Cambridge, Cambridge University Press, 1990. 
Vengono a mente, a questo proposito, le dichiarazioni d'un grandissimo matematico: "Quelques personnes ont exagéré le rôle de la convention dans la Science; elles sont allées jusqu'à dire que la Loi, que le fait scientifique lui-même étaient créés par le savant. C'est là aller beaucoup trop loin dans la voie du nominalisme. Non, les lois scientifiques ne sont pas des créations artificielles; nous n'avons aucune raison de les regarder comme contingentes, bien qu'il nous soit impossible de démontrer qu'elles ne le sont pas.» ${ }^{23}$

Infine, la riflessività degli studi sociali delle scienze su se stessi implica il doppio processo di traduzione e d'interpretazione: i ricercatori sanno quello che hanno fatto (lo studio delle rappresentazioni e delle significazioni fornite ai fatti dai laboratori e dalle reti in cui sono situati) e lo interpretano. Sulla base di questi saperi e di queste interpretazioni essi costruiscono poi i saperi e le interpretazioni degli studi sociali delle scienze. Ma ciò facendo, si mette in opera una chiusura semantica assoluta, la quale non ci permette di giudicare i procedimenti inferenziali attivati, di scoprirne gli errori, e di sottomettere i giudizi personali dei ricercatori, siano essi formali o intuitivi, agli «insight» di tipo logico. Una tale aporia rende la riflessività sospetta, poco plausibile.

\section{IL «PROGRAMMA DURO»}

Le tesi del «programma forte» sono riprese, criticate, rigettate, riformulate e radicalizzate in maniera estrema da un gruppetto di studiosi (tra i più noti, S. Shapin, M. Mulkay, K.D.Knorr-Cetina, R. Khron, R. Whitley, D. Mackenzie, T. Gyerin, ecc.) facenti capo al filosofo-sociologo Bruno Latour ed all'ingegnere-economista Michel Callon, ambedue ricercatori attivi nel «Centro di sociologia dell'innovazione» della «Scuola nazionale superiore delle miniere di Parigi», e da numerosi altri studiosi di filosofia, di sociologia e di storia, soprattutto americani e tedeschi ${ }^{24}$, collegati tra loro da due associazioni professionali (l' «European Association for the Studies of Science and Technology » e la «Society for Social Studies of Science»), ambedue molto operose sul piano editoriale, nell'organizzazione di colloqui e di congressi e nella promozione dei lavori dei loro membri ${ }^{25}$.

Contrariamente a Barnes, a Bloor ed agli altri ricercatori della Scuola di Edinburgo, questi studiosi non s'interessano ai fenomeni globali e di lungo periodo, come, per esempio, lo sviluppo dello sperimentalismo nel $\mathrm{XVII}^{\circ}$ secolo rico-

23 Poincaré, H., La valeur de la science. Avec une introduction de Louis Rougier et un portrait au pinceau de Paul Monnier, Genève, Les Éditions du cheval ailé-Constant Bourquin éditeur, 1946, p. 63.

24 Per un'informazione più precisa cfr. Felt, U., Nowotny, H., Taschwer, K., Wissenschaftsforschung. Eine Einführung, Frankfurt a.M., Campus, 1995, con bibliografia a pp. 301-318.

25 Callon, M., Défense et illustration des recherches sur la science, in Impostures scientifiques..., op. cit., pp. 253-267. Un abbozzo di giudizio d'insieme si trova in Gingras, Y., Un air de radicalisme. Sur quelques tendances récentes en sociologie de la sciences et de la technologie, «Actes de la recherches en sciences sociales », $n^{\circ} 108$, juin 1995, pp. 3-17. A questo proposito leggere le sagge e pertinenti considerazioni di Berthelot, J.-M., La science est-elle soluble dans le social? Note sur la norme du vrai et les sciences sociales, "Revue européenne des sciences sociales», XXXIV, 1996, n 104, pp. 181-186. 
struito quale prodotto d'interessi di gruppi sociali che vogliono riformare la società e resistere alle classi dominanti. I partigiani del «programma duro » studiano, invece, avvenimenti locali e di breve periodo. Al macrosociale della Scuola di Edinburgo oppongono una serie di micro-analisi limitate nel tempo e nello spazio. Per essi nessuna distinzione, empirica, analitica, epistemologica, è possibile fare tra le categorie d'oggetti ed i fattori costitutivi dell'attività scientifica. I fattori tecnici, sociali, politici ed economici sono inestricabilmente legati fra di loro. Scienza fondamentale e scienza applicata, pensiero ed azione, scienza e ricerca, sono indistinguibili, il contesto sociale determina ogni cosa, prevale sempre, determina totalmente il processo d'elaborazione della conoscenza e della diffusione e precisamente perciò non esiste alcuna differenza tra la teoria e la prassi ${ }^{26}$. La scienza è stata differenziata, per delle ragioni ideologiche, dalla ricerca e dalla tecnica. In pratica, esse si mescolano, senza alcun ordine, nell'attività quotidiana dei laboratori. Quivi i ricercatori hanno a che fare solo colla tecnoscienza.

I contenuti cognitivi (fattuali, teorici, testabili, non testabili) delle teorie scientifiche si confondono sempre con le norme dell'azione e perciò non costituiscono un sistema coerente di conoscenze, logicamente ed empiricamente, obiettive. La tecnoscienza, il cui ruolo sociale è reputato preponderante nella società postmoderna, diventa l'aspetto principale della politica contemporanea, politica, dunque, fatta con nuovi ed altri mezzi. Appunto per questa ragione tutti i fenomeni debbono essere spiegati causalmente, secondo uno schema strettamente determinista. La spiegazione causale s'applica non solo al successo ed all'insuccesso d'una teoria ma anche alla sua longevità, all'affidabilità delle macchine, ai giudizi razionali, al formalismo matematico, alla sua cumulatività, alle sue possibilità di fare delle buone predizioni, insomma ad ogni cosa. L'accettazione d'un fatto scientifico è realizzata quando tra gli attori, le forze reali in presenza e le apparecchiature materiali, in una situazione specifica, si raggiunge un punto d'equilibrio.

Latour considera determinante il ruolo degli oggetti, delle macchine, della tecnica nel lavoro scientifico. La principale attività del laboratorio è la produzione d'articoli, di relazioni, di rapporti, di comunicazioni, di perizie, di libri. Secondo questa prospettiva le «iscrizioni» (tracce, tacche, istogrammi, punti, numeri registrati, spettri, picchi ed altre cose sempre anteriori alla scrittura ma per essa tuttavia fondamentali) sono le principali unità d'analisi. Mettendo però dei «testi scritti » alla base della ricerca, la componente materiale e la tecnica, mediate dalle scritture, hanno le parvenze dell'ombra proiettata sullo schermo. Per rigettare le problematiche sollevate dalle epistemologie nomologiche e dagli approcci sociostorici, Latour considera primordiali per lo studio dello sviluppo delle scienze gli

26 Si legge a p. 219 de La science en action, op. cit., nella nota 28 di questo articolo: « Les grands initiateurs sont devenus si importants pour le modèle de diffusion que ses avocats, pris par leur propre logique maniaque, ont à dénicher qui fut réellement le premier. Cette question secondaire devient ici cruciale puisque c'est le gagnant qui emporte le tout. [...] Ceux que l'on appelle par antiphrase les «historiens des sciences » ne sont pas les derniers à ce jeu puéril qui tient plutôt de l'héraldique que de l'histoire. [...] Le mécanisme secondaire (attribution du crédit) prend le pas sur le mécanisme primaire (composition des forces).» Ed a p. 33 leggiamo: «Au lieu de considérer les aspects techniques de la science comme une boîte noire, puis de chercher quelles influence la société exerce sur eux, nous avons montré dans l'introduction qu'il était plus simple d'être là avant que la boîte se ferme et devienne noire.» 
strumenti (i dispositivi visualizzatori dei testi scientifici), i partavoce (quelli che parlano per quelli che non parlano), i laboratori (luoghi dove sono riuniti gli strumenti), gli «actants» (tutti quelli, umani e non umani che sono rappresentati). Gli strumenti, i portavoce, i laboratori e gli «actants » si trovano alla base delle reti che sottomettono lo sviluppo del fatto scientifico alla struttura d'un contesto, dei sistemi di comunicazione, degli scambi, delle modalità di valutazione e di propagazione delle idee. Le «rivoluzioni scientifiche» di Kuhn e le «falsificazioni» di Popper sono così dissolte nell'attività quotidiana delle reti e diventano delle semplici trasformazioni ( «la nouveauté d'un fait scientifique se mesure très exactement par le degré de transformation des réseaux existants »).

Alle origini di questo approccio antropologico delle scienze e delle tecniche, è consueto collocare il libro di Bruno Latour e Steve Woolgar, Laboratory Life. The Social Construction of a Scientific Fact ${ }^{27}$, monografia sul laboratorio di biologia molecolare (neuroendocrinologia) del professore Roger Guillemin, premio Nobel per la medicina nel 1978, laboratorio appartenente al «Salk Institute for Biological Studies », La Jolla, California.

In questa monografia, le dettagliate distinzioni tra le diverse categorie d'oggetti, d' « iscrizioni » e d'attori, permettono di dare un senso al reale, a quello che succede nella vita quotidiana del laboratorio. Le descrizioni e le narrazioni, rispettose dell'ordine temporale degli accadimenti, sono molto tradizionali, nonostante le professioni di fede metodologiche radicali, pretestuosamente nuove e contro-intuitive. Latour confessa d'aver vissuto due anni nel Laboratorio di neuroendocrinologia dell'Istituto Salk collo stato d'animo e nella stessa maniera dell'etnologo che lavora in una tribù primitiva di cui non sa la lingua ed i cui comportamenti gli sono estranei. Non può, quindi, fare altro se non osservare, descrivere, tentare di carpire il significato dei tentennamenti, delle esitazioni, degli sfoggi, delle tattiche, delle strategie e di tutto quello che succede nel laboratorio del professore Guillemin.

In questo ambiente, fortemente competitivo, tutto v'è concepito e percepito come realtà oggettiva, discreta, discontinua, distinta, spiegabile in termini di catene d'associazioni stabili, di «mise en réseaux». Codesta esperienza si troverebbe all'origine della sua etnografia, delle sue analisi materialistiche della produzione scientifica, ma anche del rigetto della maggior parte dei caposaldi del «programma forte» e della filosofia di Wittgestein, e sarebbe finalmente l'inizio della svolta semiologica nello studio delle scienze.

Secondo Latour e Woolgar l'attività scientifica dei ricercatori del laboratorio non ha per oggetto la natura e nemmeno mira a scoprire una realtà riposta o nascosta, preesistente. I ricercatori fabbricano, trasmettono, diffondono delle « iscrizioni », degli «statements», creano delle reti complesse ed articolate che trasformano gli enunciati in fatti ed in realtà ritenuti indubitabili, capaci di resistere alle obiezioni, alle critiche, agli attacchi dei colleghi concorrenti o rivali. Con altre parole, il lavoro scientifico consisterebbe nella fabbricazione di «attori-reti», in negoziazioni continue per costituire delle associazioni cooperative, in arruolamenti di studiosi per difendere ed imporre degli enunciati-fatti. Compito dell'ana-

27 Prima ed., Bevery Hills, Sage, 1979; 2 a ed., Princeton, Princeton University Press, 1986. Una traduzione francese corretta dal Latour è stata pubblicata a Paris, La Découverte, 1988. 
lista è di rivelare la struttura progressiva e l'organizzazione endogena di tali costruzioni. Bisogna aggiungere che la nozione di « rete» è una metafora, che Latour applica indistintamente alla scienza, alle macchine, alla scoperta, all'innovazione ed a tante altre cose.

La fabbricazione degli enunciati-fatti è descritta con minuzia: dall'ipotesi alla costruzione dell'oggetto, dalla fase sperimentale a quella del «faire-valoir» retorico, tra conflitti e gelosie personali, colle preoccupazioni di far carriera, di proteggersi dalle rivalità dei colleghi e dalle ostilità dei concorrenti. I ricercatori, dunque, non fanno altro se non fabbricare degli enunciati-fatti e renderli credibili nonostante la resistenza e l'opposizione dei colleghi ricercatori dei laboratori rivali e concorrenti. Questo lavoro di costruzione dei fatti scientifici è sostanzialmente agonistico, è un'impresa conflittuale tra agenti, oggetti, fattori tecnologici e sociali. La conflittualità condiziona, insomma, la materializzazione e l'argomentazione degli enunciati-fatti in vista di farne delle realtà esterne, le quali poi sono presunte essere antecedenti alle attività stesse della ricerca.

Tutta la realtà è per definizione sociale, secondo Latour, poiché è fatta d'associazioni eterogenee in una composizione stabile, durevole, resistente alle prove di solidità. Il sociale è, per conseguenza, un ordine di realtà distinto, dotato d'una essenza specifica, purtroppo mai definita con esattezza.

Michel Callon precisa, senza troppi infingimenti retorici, che il fatto scientifico non è né una constatazione né una testimonianza; è una costruzione elaborata e diffusa in uno spazio definito (il laboratorio e le reti che lo irrigano), al di fuori del quale non può esistere e senza il quale non può avere né presente né avvenire. Le due proprietà che caratterizzano il fatto scientifico, cioè la capacità di resistere alle critiche e la facoltà d'interessare colleghi ed utilizzatori, queste proprietà non gli appartengono in proprio, gli sono attribuite dalle negoziazioni tra gli «acteursréseaux », i soli abilitati, in verità, a dargli corpo ed a concedergli un diritto d'esistenza e di circolazione. Sono validi unicamente all'interno di queste reti di traduzione, di fabbricazione. Nonostante che questi «acteurs-réseaux» non sono né stabili né durevoli, che hanno un carattere flessibile e soggetto a modifiche di strutture, Latour e Callon li considerano costruzioni sociali. Solo disfacendo il fatto costruito da questi «acteurs-réseaux », solo effettuando la « déconstruction », si può ricostituire il modo di produzione del laboratorio e mettere in luce le reti a cui è collegato ed in cui è situato. "La fabrication des faits est un processus continu, qui consiste en la mobilisation progressive, tâtonnante et négociée de réseaux auxquels ils doivent in fine leur robustesse et la mesure de leur universalité (relative). La séparation entre création et objectivation n'est possible qu'après coup, lorsqu'il s'agit de donner un nom et un statut à une opération réussie. ${ }^{28}$

Un fatto scientifico ha sempre la forma che gli danno i canali di comunicazione, le interconnessioni, i collegamenti integrati, le reti che l'alimentano; un fatto scientifico non è il risultato d'una decisione razionale presa da uno spirito libero e senza legami di parte, è il prodotto d'una o parecchie reti sociotecniche, è impuro per natura poiché è composto d'elementi eterogenei provenienti, per caso,

Callon, M., Introduction, in: Sous la direction de Michel Callon, La science et ses réseaux. Genèse et circulation des faits scientifiques, Paris, Editions de la Découverte, Strasbourg, Conseil de l'Europe, Paris, Unesco, 1989, pp. 25-26. 
di qui e di là. Vive in quello spazio pubblico chiamato rete sociotecnica e proprio perciò deve resistere alle critiche e suscitare l'interesse.

La conoscenza scientifica è in questo modo ridotta ad un processo attraverso il quale si dà un significato a coefficienti scientifici ed extrascientifici, ad ideologie, ad intrighi accademici, insomma a tutte le componenti d'una attività sostanzialmente sociale. Dal momento che la natura e lo spirito umano hanno la stessa origine, la loro separazione e la loro differenziazione avviene soltanto nella tappa finale del processo costruttivo. Anche l'efficacia del fatto è d'origine sociale: è il risultato di concatenamenti, di legami, di reti, di strategie di controllo. Siccome un insieme d'operazioni comporta risposte identiche, non c'è ragione di stupirsi se con lo stesso test s'ottengono gli stessi risultati, in California ed in Arabia Saudita. La stabilizzazione d'un fatto e la sua efficacia operativa sono le conseguenze e non la causa dell'attività scientifica, quindi essi sono indifferenti alla realtà esterna ed alle metodologie impiegate. Fare scienza significa costruire e difendere punti di vista funzionali trasformabili talvolta in oggetti stabilizzati, creare l'ordine a partire dal disordine. Siccome non esistono valori universali, lo scienziato deve cambiare continuamente il suo punto di vista per stabilire delle relazioni tra mondi incommensurabili. Grazie a questa forma di relativismo Latour può di nuovo riaffermare che il carattere sociale della scienza e della tecnica scaturisce dalle strategie di costruzione e di stabilizzazione delle catene d'associazioni.

Ovviamente, da una siffatta concezione deriva senz'altro che «fatti » ed « artefatti » (cioè gli effetti parassiti prodotti da una causa secondaria non controllata dal metodo o dall'osservazione e di cui l'esperimentatore non è cosciente) si differenziano solo per la presenza o per l'assenza della referenza al processo di costruzione. Tra di loro non esiste alcuna disparità di sostanza.

In breve, i partigiani del «programma duro » studiano la scienza in azione, nel momento in cui è fatta anziché la scienza già fatta, quella che è stata liberata dalle manipolazioni del processo produttivo. Questa scienza in azione ignora le discussioni sulla corrispondenza fra gli enunciati ed il mondo, fra i discorsi e la realtà, fra il ruolo dell'esperienza e l'interpretazione dei risultati. Le qualità intrinseche dei costrutti scientifici (funzioni referenziali, coerenza, semplicità, inserzione in contesti più vasti, fecondità, utilità, ecc.) sono ritenute meno significative delle trasformazioni che hanno dovuto subire per accedere alla stabilizzazione della loro esistenza. La contestualizzazione diventa un principio esclusivo di determinazione. La soluzione apportata ad una controversia non ha niente a che fare collo stato di natura e nemmeno coll'assetto durevole della società, è sempre ed unicamente la causa, mai la conseguenza, d'un enunciato o d'una rappresentazione stabilizzati. Stato di natura e stato della società sono le conseguenze simmetriche dell'azione incerta e titubante dei ricercatori per reclutare umani e non-umani, per dividere l'esterno dall'interno, per formare le tecnoscienze. Lo studio degli errori logici e delle distorsioni indotte dalle strutture della Società è sostituito da quello che ricostruisce «l'angle et la direction du déplacement de l'observateur et la longueur du réseau qui se construit ainsi ». «Avant d'attribuer une qualité particulière à l'esprit ou à la méthode scientifique, nous examinerons d'abord les nombreuses façons dont les inscriptions sont regroupées, combinées, liées entre elles et renvoyées. C'est seulement si quelque chose reste inexpliqué une fois que nous aurons étudié les réseaux longs que nous pourrons commencer à parler de facteurs cognitifis ». 
Tutto ciò vuol dire che lo studioso non è mai confrontato alla natura. Se essa ha un ordine, quest'ordine è un ordine imposto, frutto d'una contingenza storica, risultato d'un processo di negoziazioni. Quest'ordine, arbitrario ed artificioso, è l'effetto d'una gamma d'associazioni, più o meno forti, di umani e di non umani. La storia di queste associazioni è la storia della tecnoscienza, cioè la storia delle risorse accumulate lungo le reti per accelerare l'iscrizione, la mobilità, l'affidabilità, la capacità di combinazione e la coesione delle tracce che rendono possibile anche l'azione a distanza $^{29}$. Tra il capire cosa siano i fatti, quali le funzioni delle macchine, chi siano gli addetti ai lavori ed il perché ed il come delle loro intenzioni e dei loro progetti, per Latour non c'è nessuna differenza. Tra la costruzione della scienza, il carattere agonistico della stessa, la materializzazione o reificazione del fatto scientifico, la sua credibilità, tra la scienza prodotto di circostanze specifiche e locali, credibile ed esistente se presente costantemente sul mercato dell'informazione (l'assenza favorisce gli avversarsi ed i concorrenti), esiste, secondo Latour, una relazione causale d'ordine meccanico. Quando il Laboratorio Alfa mette sul mercato dei beni simbolici degli enunciati che discreditano tutti gli altri enunciati contenenti meno informazioni, la credibilità del Laboratorio Alfa cresce necessariamente e quella di tutti gli altri diminuisce. Se la forza di convinzione degli enunciati di questo Laboratorio Alfa è robusta, allora gli altri laboratori cesseranno persino di muovere delle obiezioni; lo statuto di fatti, di cose obiettive reali, di cui non si può più mettere in dubbio l'esistenza, è acquisito in modo conclusivo ed il successo è perciò assicurato.

Se nell'attività scientifica, il sociale ed il tecnico si confondono, se è impossibile distinguere i fatti dagli artefatti, i fattori esterni dai fattori interni, il discorso del senso comune dal ragionamento scientifico, gli scienziati in quanto tali sono necessariamente plasmati da questa competizione, dai conflitti d'appropriazione che hanno luogo nel laboratorio e tra i laboratori ${ }^{30}$.

Secondo Latour, lo scienziato costruisce «l'oggetto scientifico», ma per far credere alla realtà esterna deve ingegnosamente mascherare le operazioni di costruzione. Deve praticare con talento l'illusionismo, ha per unica ambizione quella di far credere alle illusioni che pratica, ma è anche vittima delle sue proprie

29 Latour, B., Science in Action. How to Follow Scientists and Engineers through Society, Cambridge, Harvard University Press, 1987 e l'edizione francese rivista: La science en action. Introduction à la sociologie des sciences, Paris, Gallimard, 1995, dove a pp.627-630 si trovano espresse sotto la forma di tesi le regole di metodo ed i principi ispiratori del «programma duro». Delle applicazioni di questi principi metodologici sono presentate in Petites leçons de sociologie des sciences, Paris, La Découverte, 1993, ed in maniera chiara e compendiosa per i non addetti ai lavori nei due libretti: Le métier de chercheur. Regard d' un anthropologue, Paris, INRA, 1995 e Petite réflexion sur le culte moderne des dieux faitiches, Le Plessis-Robinson, Synthélabo, 1996, ed in Pandora's Hope. An essay on the Reality of science studies, Cambridge, MA, Harvard University Press, 1999, tr. fr., Paris, La Découverte, 2001. Ved. a questo proposito Science wars. Phony Peace, «The Economist», October 16th 1999, pp. 10-11.

30 Cassier, M., Le partage des connaissances dans les réseaux scientifiques: l' invention de règles de «bonne conduite» par les chercheurs, «Revue française de sociologie», XXXIX, 1998, n 4, pp. 701-720, mostra molto bene che nelle « reti scientifiche» la competizione ed il conflitto non intaccano le regole di gestione della conoscenza, che i ricercatori arrivano a conciliare il «comunalismo» con gli interessi individuali, la pubblicità dei risultati ottenuti con il segreto voluto dai finanziatori della ricerca. Le comunità scientifiche sanno fare emergere delle nuove regole, dei nuovi diritti, delle nuove maniere di gestire le conoscenze, che non tengono conto dell'agonismo di cui tanto enfatizza il «Programma duro». 
illusioni. Curiosamente, il fatto appare nel pensiero dello scienziato dopo essere stato concepito dai dispositivi sperimentali, fabbricato dalle macchine, dalle circostanze, dalle risorse disponibili, ecc. ecc. Non c'è una realtà esteriore, bisogna costruirla, metterla in ordine, renderla credibile ed in pari tempo postularla come se fosse effettivamente presente. Per conseguenza, il ricercatore vive costantemente nell'illusione. Tutto è illusione, la sola realtà è la costruzione, ma tutti, persino gli stessi costruttori, continuano a credere nella realtà esteriore, vittime di quest'illusione da essi stessa fabbricata coll'ausilio di macchine, d'oggetti, d'animali e di diverse specie di quasi-oggetti e di ibridi.

Ma se tutto è illusione, come mai certe illusioni durano così a lungo e sono atte a risolvere tanti enigmi intellettuali e tante difficoltà pratiche? Se la scienza ha le sue fondamenta ed i suoi contenuti nella società e nelle sue istituzioni, come mai degli individui isolati ed indipendenti possono fabbricare dei fatti scientifici senza legami colla realtà sociale e talvolta in contraddizione con essa?

Ammettiamo che Louis Pasteur, tra il 1870 ed il 1914, abbia reso convincenti e credibili le sue dottrine grazie a delle associazioni, che la profilassia della rabbia e l'asepsi si siano imposte a causa delle alleanze che lo scienziato era riuscito a stabilire con gli igienesti, cogli addetti ai lavori in batteriologia, coi politici desiderosi d'uno sviluppo sanitario rapido e d'una modernizzazione della società francese. Possiamo inferire da tutto ciò che le distinzioni scienza/tecnica, società/ natura, dimensione politica della scienza/verità della stessa scienza, rapporti logici/rapporti di forza, siano stati degli espedienti retorici ed in più che Pasteur e tutti quelli che ne hanno accettato le dottrine siano stati succubi, per dirlo colle parole stesse di Latour, di «croyance dans l'existence du monde moderne, croyance dans l'existence de la logique, croyance dans la présence de la raison, croyance aussi dans la croyance elle-même et dans le savoir tout aussi bien » $?^{31}$ C'è da dubitarne fortemente.

Che la natura non parli da sola, che i fatti scientifici siano costruiti, che la conoscenza non sia una fotografia o una registrazione dei risultati forniti dall'esperienza, sono dati che la filosofia della scienza e le epistemologie interne delle diverse discipline hanno sempre accettato. Che la scienza sia immersa nella storia e nella società è un dato di fatto incontestabile; che l'enunciato scientifico debba fare i conti colle tecnologie disponibili, con i dispositivi materiali, istituzionali,

31 Una presentazione magistrale delle dottrine di Latour è stata fatta da Isambert, Fr.-A., Un «programme fort» en sociologie de la science?, «Revue française de sociologie », XXVI, 1985, $\mathrm{n}^{\circ} 3$, pp. 485-508 e dello stesso Après l'échec du «programme fort», une sociologie du contenu reste-telle possible?, in Boudon, R. \& Clavelin, M. Ed., Le relativisme est-il résistible? Regards sur la sociologie des sciences. Actes du Colloque international «La sociologie de la connaissance scientifique: bilan et perspectives», Université de Paris-Sorbonne, 21-22 janvier 1993, Paris, PUF, pp.51-76. Degli scritti sul Latour citiamo soltanto: Queré, L., Les boîtes noires de B. Latour ou le bien social de la machine, «Réseaux», n³6, juin 1989, pp. 96-117; Borraz, O., La science estelle une sociologie?, «Politix », $\mathrm{n}^{\circ}$ 10-11, 1990, pp. 135-144; Chateauraynaud, F., Forces et faiblesses de la nouvelle anthropologie des sciences, «Critique», $\mathrm{n}^{\circ}$ 529-530, juin-juillet 1991, pp. 459-478; Bourdieu, P., Raisons pratiques. Sur la théorie de l' action, Paris, Seuil, 1994, pp.91-97 e Les usages sociaux de la science. Pour une sociologie clinique du champ scientifique, Paris, INRA, 1997; Ragounet, P., D’une critique sociologique des épistémologies positives à l'abandon du projet sociologique, «Revue suisse de sociologie», 20, 1994, n² 2, pp. 487-503; Brown, J.R., La science prosaïque de Bruno Latour, in: Sous la direction de Kremer-Mariotti, A., Sociologie de la science, op. cit., pp. 151-170. 
sociali e cognitivi adoperabili, è un'affermazione scontata, banale. Tutto diviene però più complicato se si sostiene che non c'è alcuna differenza tra il momento della creazione e quello della giustificazione ${ }^{32}$, quando si postula che la pratica scientifica è garantita esclusivamente dal principio di simmetria (trattare parimenti il vero ed il falso, la natura e la società, il sociale ed il tecnico, gli oggetti animati e gli oggetti inanimati, gli uomini e le macchine) e mai dall'analisi logica e dalla verificazione empirica.

Non si sa per quali ragioni la scienza che si fa sia più importante della scienza già fatta, non si sa bene perché la vera scienza sia quella che emerge dalle modalità della sua produzione. In verità, l'analisi del processo di fabbricazione d'una teoria non ci dice nulla sulla validità finale e sull'efficacia della stessa. Questa validità è assicurata soprattutto dalla coerenza logica, dalla conformità alla realtà sperimentale, dal tempo, dalla durata e da individui che vivono ed operano, in un contesto mutato, molti anni dopo la costruzione scientifica.

Latour rifiuta di trattare asimmetricamente attività il cui statuto è diverso perché ritiene che non disponiamo d'una misura che ci permetterebbe d'accettare una tesi e di rifiutarne un'altra, di spiegare come si sia giunti alla decisione che attribuisce la vittoria alla teoria $A$ a scapito di tutte le altre.

Una tale tesi sarebbe sicuramente pertinente se agissimo in uno spazio vettoriale e se disponessimo d'una dimostrazione grazie alla quale l'échec ed il successo, il logico ed il non logico, potrebbero essere comparati.

Questa etnografia della scienza non produce che delle eccellenti descrizioni del lavoro nel laboratorio e da esse, particolari e contingenti, Latour ricava, grazie a degli espedienti retorici, una filosofia ipercritica della modernità. È vero, la società moderna, per rendere ordinate le pratiche umane, ha elaborato una serie di coppie filosofiche, d'opposizioni formali, d'asimmetrie, di saperi pratici e di saperi teorici, come per esempio le dicotomie natura/società, oggetto/soggetto, locale/globale, unità/diversità, moderno/tradizionale, permanenza/cambiamento, trascendenza/ immanenza, uguaglianza/ineguaglianza, insieme continuo/insieme discreto, centrato/acentrato, dipendenza/indipendenza, e così di seguito. Latour dà a questa opposizione un'importanza strategica decisiva. Per lui questa nostra società produce di continuo altre dicotomie, altre opposizioni, altre asimmetrie, altri saperi, ma anche le condizioni per denunciarli e criticarli. Nel momento stesso in cui produce queste asimmetrie, mette in pratica degli espedienti per mescolare, complicare, tenerle insieme quali simmetrie e per ottenere il meticciamento di uomini e cose. Ciò facendo la Società occidentale dimostra di non tenere nessun conto del suo proprio modello teorico con pretese universaliste. L'inquinamento, gli embrioni, i virus della AIDS, i robot intelligenti, l'informatica, tante macchine ibride, le protesi umane capaci di captare le onde elettromagnetiche, e tanti altri ibridi, fanno parte della nostra vita quotidiana e purtuttavia, continua Latour, noi continuiamo a privilegiare come uniche ed esclusive le attività degli essere umani.

Se vogliamo liberarci di questo paradosso e di tutte le conseguenti aporie non ci rimane che una via d'uscita: rinunciare ad essere moderni: «Nous n'avons

32 Vedere a questo proposito l'eccellente messa a punto di Petroni, A. M., Conventionalism, scientific Discovery and the sociology of Knowledge, «International Studies in the Philosophy of Science», 7, 1993, n³, pp. 225-240. Un'altra versione di questo scritto si trova in Le relativisme est-il résistible?, op. cit., pp.101-125. 
guère le choix. Si nous ne changeons pas la maison commune, nous n'y absorberons pas les autres cultures que nous ne pouvons plus dominer et nous serons jamais incapables d'y accueillir cet environnement que nous ne pouvons plus mâ̂triser. Ni la nature ni les Autres ne deviendront modernes. C'est à nous de changer nos façons de changer ${ }^{33}$.

Pretesa luciferina, miraggio filosofico, speranza intellettuale? Ai posteri l'ardua risposta.

Che il filosofo, lo storico ed il sociologo debbano interessarsi alla vita del laboratorio mi sembra un compito ragionevole ed inderogabile. Ma perché ridurre tutto quello che succede e si osserva nel laboratorio ad un solo fattore, quello di fabbricare degli enunciati e di metamorfizzarli in fatti? Perché assimilare la distribuzione delle competenze tra la scienza e la politica alla controversia sulla pompa pneumatica tra Thomas Hobbes e Robert Boyle? Perché negare l'autonomia relativa di certe opere dell'attività umana, perché ridurle al sociale concepito come strategie d'associazioni e quindi come rapporto di potere?

Se le identità e le differenze sono prodotte e costruite da attori all'interno di laboratori inseriti in reti, perché mai tutti accettiamo delle nozioni e dei concetti, da secoli in circolazione, come scienza e tecnica, come protone e neutrone, come genotipo e fenotipo e così di seguito? Se la costruzione d'un enunciato si fa in una rete e mediante una rete, se è la risultante d'un successo locale, spiegabile in termini di rapporti di forza particolari e momentanei, com'è allora possibile che diventi, sul lungo periodo, parte integrante del nocciolo duro del sistema scienza? Come spiegare il fatto che certe teorie funzionano in situazioni diverse ed anche in contesti congiunturali discontinui? Perché la verità d'una teoria non sempre coincide colla sua efficacia? Per quali ragioni dubitare del rapporto esistente tra le concettualizzazioni degli uomini ed il controllo che grazie ad esse è assicurato agli umani sul mondo esteriore? Perché non riconoscere che le finalità delle pratiche umane sono disparate e differenti? Le finalità dell'astronomia e della botanica, per esempio, sono diverse da quelle dell'astrologia e della stregoneria.

Latour e Callon attribuiscono al lavoro scientifico una finalità: quella di controllare il mercato dei beni simbolici scientifici. Essi riducono la validità delle proposizioni della scienza alla loro credibilità e questa a puri rapporti di forza. I ricercatori diventano così gli strategi d'una competizione in cui la sola posta in gioco importante è lo sbaraglio dei concorrenti, rendere innocui i rivali, controllare l'incertezza inerente all'accumulazione, alla circolazione, alla trasformazione di questo capitale importantissimo che è ormai la scienza nella società moderna.

Per essere coerenti colla tesi che non esistono criteri universali per la validazione delle proposizioni, dobbiamo accettare che anche questa stessa tesi non è universale, che è relativa. Se ammettiamo che tutte le teorie hanno dei valori equivalenti, non possiamo nemmeno negare la pertinenza dell'affermazione che tutte le teorie non hanno lo stesso valore. La validità del relativismo non può essere fondata sul relativismo stesso, deve far ricorso a principi non relativisti, assoluti, universali. Cambiare punti di vista e stabilire relazioni tra mondi incommensurabili è possibile ma a condizione di disporre di categorie inglobanti, cioè universali.

33 Latour, B., Nous n'avons jamais été modernes. Essai d'anthropologie symétrique, Paris, La Découverte, 1991, p.198, ed in maniera ancora più radicale in Politiques de la nature. Comment faire entrer les sciences en démocratie, Paris, La Découverte, 1999. 
Studiosi come Collins e Pickering ${ }^{34}$ hanno maggiorato le caratteristiche relative e sociali delle scienze sottolineando la contingenza dei loro risultati e mettendo in rilievo il fatto che lo scienziato, quando inizia una ricerca, parte da un certo numero di presupposti, di conoscenze di base, d'abilità, in vista di realizzare un certo fine. Il suo lavoro costituisce una sequenza caratterizzata da tentativi, da prove, da errori. Deve scegliere, continuamente, tra diverse strategie possibili: continuare, rinunciare, ripartire da capo, modificare la strumentazione, rivisitare la teoria, ricercare nuove informazioni, modificare il protocollo di ricerca, ecc.ecc. Quando si sa ciò che si cerca, la situazione è relativamente semplice. Ma se non lo si sa, allora tutto è più complicato perché non si sa se l'effetto osservato sia o non autentico, se bisogna ritenerlo, chiudere l'esperimento, rifarlo per essere sicuri di non avere a che fare con un artefatto, se è opportuno modificare la teoria, rigettarla o ancora rimetterla alla prova. Da questo punto di vista la ricerca è caratterizzata da un complesso di decisioni prese in situazioni d'incertezze. Non esistono regole che indichino come procedere in qualsiasi circostanza. Le scelte dello scienziato non sono guidate né dalla teorie né dal metodo. Sono prese in maniera arbitraria, sulla base di precostruiti sociali. Appunto perciò la sperimentazione non risolve i problemi, non discioglie le controversie, non scombina le concettualizzazioni.

La Scuola di filosofia delle scienze di Stanford non rigetta tutte le dottrine del «programma duro », quelle che negano l'esistenza d'una razionalità universale, d'un metodo scientifico in misura di collegare deduttivamente gli enunciati osservazionali singolari a leggi universali, ma modifica certe parti di quelle dottrine. I membri della Scuola di Stanford riconoscono, infatti, che le teorie nelle scienze hanno delle funzioni di rappresentazione e d'intervento, più o meno affidabili, più o meno simboliche, ed a ragione essi sottolineano che esistono numerosi stili di ragionamento, diversi livelli teorici, che le scienze sono più o meno efficaci, hanno cioè la capacità d'ottenere l'effetto programmato. Le scienze ci permettono di manipolare la realtà e di controllarla con mezzi indipendenti dai fattori sociali e politici. Esse sono eterogenee, costituite da modelli teorici e fenomenologici collegati tra di loro in maniere diverse, disparate, e non sempre rigorosamente, e talvolta formano dei sistemi cognitivi e socio-tecnici «bricolés», come direbbe Claude Lévi-Strauss, e la cui ricostruzione razionale è assai complicata e complessa ${ }^{35}$.

34 Collins, H. M., Sociology of Scientific Knowledge, Bath, Bath University Pres, 1982 e Changing Order. Replication and Induction in Scientific Practice, London, Sage, 1992; Pickering, A., The Mangle of Practice. Time, Agency and Science, Chicago, University of Chicago Press, 1995 e dello stesso Beyond Constraints. The Temporality of Practice and the Historicity of Knowledge, in Buchwald, J.(Ed.), Scientific Practice, Chicago, University of Chicago Press, 1995.

35 Per una prima approssimazione su questa Scuola, si rimanda a Cartwright, N., How the Laws of Physics Lie, Oxford, Clarendon Press, 1983 e dello stesso Nature's Capacities and their Measurement, Oxford, Oxford University Press, 1989, ma anche a Hacking, I., Representing and Intervening. Introductory Topics in the Philosophy of the Natural Sciences, Cambridge, Cambridge University Press, 1983; a Suppe, F., Probabilistic Metaphysics, Oxford, Blackwell, 1984 ed a Dupré, J., The Desorder of Things, Harvard, Harvard University Press, 1995. La posizione di questa scuola è, per certi versi, non diversa da quella di Benedetto Croce, per il quale la scienza, per il suo carattere pratico, economico, insomma per la sua efficacia, ci consente una potenza sul mondo superiore a tutti i saperi, ed in quanto tale è diversa dalla religione, dalla stregoneria, dalla magia, ecc. Per Croce la scienza non è però «conoscenza», per la Scuola di Stanford invece è la migliore forma di cognizione e gerarchicamente il sapere più elevato. 
Che tutte queste ricerche siano caratterizzate da un relativismo più o meno radicale, i cui confini restano sempre incerti, è un fatto indiscutibile. Che questo relativismo cognitivo, attento alla variabilità dei significati e degli impieghi dei concetti scientifici, ai contesti locali, pronto a contestare dualismi, distinzioni e gerarchie tra scienza ed ideologia, natura e cultura, fatti e teorie, verità e falsità, soggettività ed oggettività, scienza e senso comune, - che questo relativismo sia una critica della ragione, del razionalismo, del realismo, che sia un'apologia del differenzialismo e del multiculturalismo, una critica della modernità, non esiste il minimo dubbio. Ed è proprio per questa sua portata ideologica che ha esercitato ed esercita un'influenza considerevole nei più diversi settori della cultura ${ }^{36}$.

\section{L' «AFFARE SOKAL»: BEFFA O IMPOSTURA?}

È notorio che da qualche lustro le Università americane sono agitate da scontri intellettuali d'una intensità e d'una rudezza senza pari. Le frontiere tra le discipline e le «querelles» ideologiche sono state rimescolate; l'utilità immediata è divenuto il criterio legittimante tutte le attività scientifiche. Una controcultura accademica s'è costituita e si è attribuita la missione di far riconoscere dalla Società i particolarismi, le differenze, di riabilitare e di conservare ciò che struttura le identità delle minoranze etniche, del «gender», del femminismo, del lesbismo e dell'omosessualità maschile. La civiltà moderna è giudicata eterofobica, ragion per cui bisogna combatterla in nome del multiculturalismo localista, del differenzialismo, del femminismo, del radicalismo ecologico, dell'attivismo AIDS e del post-modernismo. Secondo questa controcultura, le scienze consolidano l'eterofobia, sono al servizio di interessi militari ed industriali antidemocratici, restano le paladine più forti del conservatorismo, dell'androcentrismo, delle sedicenti leggi del mercato. I «Chicaños Studies », i «Gay and Lesbian Studies », i «African-American Studies » e tanti altri «X-Studies», aggiungono alla loro contestazione del realismo, alla loro difesa del relativismo anche degli obiettivi morali e politici nonché la rivendicazione delle loro differenze d'identità ${ }^{37}$. Sandra Harding, una dei leader dei «Women's Studies », per esempio, non esita a proclamare ed a scrivere che la scienza occidentale è antifemminista, esclude le donne dalla ricerca scientifica, le relega nella sfera domestica, e precisamente per tutte queste ragioni bisogna costruirne un'altra femminista, antirazzista, capace di rivelare i precostruiti impliciti che fissano i domini disciplinari, le metodologie, le deontologie, i discorsi e gli scritti sull'ordine naturale, sulla razionalità, sull'oggettività, precostruiti spacciati per evidenze e che hanno invece il compito di dis-

36 Un'accurata e succosa presentazione delle controversie che sono alla base della corrente culturale del «post-modernismo» è stata pubblicata recentemente in italiano da Lolli, G., Beffe, scienziati e stregoni. La scienza oltre realismo e relativismo, Bologna, Il Mulino, 1998.

37 Basti qui rimandare per una prima informazione a quella data da critici di questo processo di radicalizzazione politica della vita accademica in particolare e della vita culturale in generale, Bloom, A., The Closing of the American Mind, New York, Simon and Schuster, 1987, ed a Jacoby, R., The last Intellectuals. American Culture in the age of Academy, New York, Basic Books, 1987. 
simulare il sessismo, il razzismo, le ingiustizie, e di mistificare così i veri principi democratici e libertari ${ }^{38}$.

La critica culturale e la contestazione radicale delle controversie scientifiche sull'oggettività e sulla razionalità diventano la struttura portante d'un impegno politico per la realizzazione del progetto di democratizzazione egualitaria dei rapporti sociali ${ }^{39}$. I «Cultural Studies of Science», i «Social Science Studies», i «Science-Technology-Society Studies», pretendono che la scienza è una costruzione sociale, che la verità scientifica dipende dall'interpretazione soggettiva di coloro che si sono imposti come gli specialisti della materia. La «testualità » costruisce socialmente il mondo reale. Anche quando ammettono che il mondo reale è indipendente dalle nostre osservazioni, gli studiosi appartenenti a questa corrente intellettuale pretendono che le interpretazioni elaborate sono plasmate dalla nostra origine sociale, dalla formazione ricevuta, dai valori della classe d'appartenenza. Insomma, il sistema concettuale che utilizziamo, non esprime mai verità oggettive. La differenza tra la scienza ed il mito sussiste, è comprensibile se rapportata alla modernità occidentale, è valida se legittimata da questa tradizione, altrimenti è infondata, e non è niente altro che una formula ideologica.

Stanley Aronowitz e Andrew Ross, con grande perseveranza, tentano di persuadercene e di farci credere che la verità, l'evidenza, l'argomentare sono presupposti, pregiudizi, valori di classe, i quali celano, in realtà, duri rapporti di potere ed assicurano l'egemonia d'un gruppo sociale su tutti gli altri ${ }^{40}$.

Le reazioni da parte degli studiosi di scienze esatte contro $i$ «Cultural Studies of Science» sono, ovviamente, durissime e tra le più argomentate annoveriamo quelle del biologo Paul R. Gross, del matematico Norman Levitt e del geografo Martin W. Lewis, promotori di colloqui, di dibattiti, di tavole rotonde, e poi di due importatissimi volumi diffusi ampiamente ed all'origine di virulente controversie presto tracimate sulla stampa periodica e sugli schermi $\mathrm{TV}^{41}$.

Agli inizi dell'autunno del 1994, un professore di fisica teorica, Alan Sokal, dell'Università di New York, militante sandinista della sinistra terzomondista, invia alla redazione portabandiera degli studi culturali post-modernisti statunitensi un articolo con un titolo reboante (Violare le frontiere : verso un' ermeneutica tras-

38 Sull'argomento sono da leggere le pacate, finissime considerazioni di Feldman, J., Femme, féminisme, science, in «L'Année sociologique», 42, 1992, pp. 425-440. Della stessa ved. le stringate osservazioni sull'intera controversia: Is there a post-modern science? The Signification of the Sokal Affair, in «Philosophy and Social Action», 25, 1999, n. 3, pp. 19-30.

39 Harding, S., The Science Question in Feminism, Ithaca, N.Y., Cornell University Press, 1986; Whose Science? Thinking from Women's Lives, Ithaca, N.Y., Cornell University Press, 1991; Science is «Good to think with», «Social Text», 14, Spring-Summer 1996.

40 Ved. a questo proposito Aronowitz, S., Science as Power. Discourse and Ideology in Modern Society, Minneapolis, University of Minnesota Press, 1988; Aronowitz, S. \& Giroux, H., Postmodern Education: Politcs, Culture and Social Criticism, Minneapolis, University of Minnesota Press, 1989; Ross, A., Strange Wheater. Culture, Science and Technology in the Age of Limits, London, Verso 1991.

41 The Fligt from Science and Reason. Edited by P. R. Gross, N. Levitt, and M. W. Lewis, Baltimore, The Johns Hopkins University Press, 1996 e 1997, e Gross, P. R. \& Levitt, N., Higher Superstition. The Academic Left and Its Quarrels with Science, Baltimore, The John Hopkins University Press, 1994 e 1998. Quest'ultimo libro ha suscitato aspre polemiche. Citiamo soltanto Martin, B., Social Construction of an «Attack on Science», «Social Studies of Science», 26, 1996, pp. 161-173. 
formatrice della gravità quantica), tutto incentrato sulle implicazioni filosofiche, politiche, sociali delle teorie fisiche del $\mathrm{XX}^{\circ}$ secolo, pieno di citazioni d'autori americani e francesi alla moda, di riferimenti alla politica, alla sociologia, alla filosofia, alla psicanalisi. Anche i passi in cui si parla di matematica e di fisica sono bizzarri. L'articolo, corredato da 218 riferimenti bibliografici e da ben 109 lunghe note, è però sprovvisto di prove, è infarcito di controsensi, è zeppo di riferimenti inventati a strane teorie ed a scritti, spudoratamente travisati, d'autori notissimi.

I cinque redattori di «Social Text» domandano all'autore di raccorciare il testo, di sopprimere alcuni sviluppi filosofici ritenuti fuori moda, di tagliare un certo numero di note e di riferimenti bibliografici. Sokal oppone un rifiuto e lo giustifica coll'argomento che l'articolo è stato redatto soprattutto per far capire ai suoi colleghi fisici quanto siano importanti gli studi culturali per il progresso della fisica. La redazione, ingaggiata nelle polemiche alimentate dall'offensiva di Gross e Levitt, lusingata che un fisico le sottomettesse un articolo in difesa degli studi culturali attaccati dai più illustri rappresentanti delle scienze dure, decide, dopo molteplici trattative ed esitazioni, di inserirlo in un numero speciale della rivista consacrato alla «Guerra delle scienze», numero speciale che esce nel mese di maggio $1996^{42}$. Poche settimane dopo l'uscita della rivista, Sokal fa sapere che l'articolo pubblicato è un «esperimento », una parodia scritta coll'intento di provare l'inconsistenza, la superficialità delle dottrine difese dai partigiani delle filosofie post-moderniste, la loro ignoranza delle dottrine scientifiche, di cui però discettano con dilettantesca improntitudine ${ }^{43}$.

Cosa, in poche parole, Sokal dice in quest'articolo? Lo scritto comincia con una critica virulenta degli scienziati che restano fedeli ai dogmi della filosofia dei Lumi, imposti con «violenza simbolica» a tutta la cultura occidentale. Per questi studiosi credenti nella filosofia illuministica, esisterebbe un mondo esterno le cui proprietà sono indipendenti dall'uomo. Costui potrebbe ottenerne una conoscenza affidabile, sia pure imperfetta e approssimativa, purché si conformasse alle procedure oggettive ed alle esigenze epistemologiche prescritte dal sedicente metodo scientifico. La ragione sarebbe lo strumento principale per appropriarsi concettualmente della realtà ed anche per controllarla.

Lo scritto di Sokal proclama che siffatta pretesa è sprovvista di basi solide e di validi motivi. Infatti, le teorie della relatività generale e della meccanica quantistica hanno dimostrato che persino la fisica è una costruzione sociale e linguistica, come del resto i premi Nobel Niels Bohr e Werner Heisenberg hanno già sovente affermato.

42 Sokal, A., Transgressing the Bounderies: Toward a Transformative Hermeneutics of Quantum Gravity, «Social Text», $\mathrm{n}^{\circ}$ 46-47, Spring-Summer 1996, pp.217-252. La traduzione francese si trova nel libro di Sokal, A. e Bricmont, J., Imposture intellectuelles, Paris O. Jacob, 1997, pp. 211 252. A carte 253-260 dello stesso libro si trovano dei commentari sulla parodia. Alan Sokal e Jean Bricmont hanno pubblicato alla fine del mese di marzo 1999 a Parigi, nella collezione «Le livre de poche» una seconda edizione del loro libro Impostures intellectuelles, con una lunga prefazione e moltissime aggiunte e nuovi complementi. I due autori rispondono anche a tutte le critiche che il libro ha suscitato e forniscono importanti precisazioni sulla natura delle stroncature di cui stati ingenerosamente avviliti.

43 Sokal, A., A Physicist Experiments with Cultural Studies, «Lingua Franca», May-June 1996, pp. 62-64. 
Sokal continua sostenendo che la gravitazione quantistica conferma le confutazioni post-moderne dell'oggettività e della verità, pone le premesse d'una scienza fisica «liberatrice» al servizio delle cause politiche progressiste. Poi ricorre alla fisica dell'infinitesimale, a delle strane operazioni di derivazione e d'integrazione, a delle funzioni lineari (ove però non vi sono né incognite né variabili di primo grado) e discontinue, per dimostrare la fondatezza delle conclusioni politiche dei «Cultural Studies».

Siccome per rendere conto d'un oggetto unico è necesario ricorrere a diversi punti di vista, Sokal aggiunge: «Come e perché, in una tale situazione, il clero secolare degli scienziati che godono credito e fiducia e che s'auto-perpetuano, come e perché questo clero secolare possa pretendere di conservare il monopolio sulla validazione del sapere scientifico? [...] Il contenuto e la metodologia della scienza post-moderna forniscono un sostegno intellettuale importante al programma politico progressista, inteso nel senso più lato: la trasgressione delle frontiere, la rottura delle barriere, la democratizzazione radicale di tutti gli aspetti della vita sociale, economica, politica e culturale.»

In conclusione di questa parte dell'articolo, Sokal fa un'escursione nel territorio delle nuove matematiche per constatare che la teoria standard degli insiemi di Zermelo-Fraenkel sta per essere, finalmente, abbandonata. Le nuove matematiche non irregimentano più con leggi e teoremi, con strutture algebriche costrittive, le aspirazioni progressiste e post-moderne della fisica nuova che sta elaborandosi.

In tutte le pagine e in tutte le note dell'articolo si trovano delle cose strampalate: i campi della teoria dei quanti confermano le dottrine psicanalitiche sulla natura del soggetto nevrotico di Jacques Lacan, le logiche paraconsistenti i programmi politici della sinistra, il teorema di Bell sui fondamenti della meccanica quantistica viene ad essere la copertura del capitalismo industriale, l'assioma di Zemerlo è un ostacolo al femminismo e alla libertà dell'aborto, la costante geometrica $P i$ d'Euclide e la costante $G$ di Newton perdono la loro universalità, diventano variabili inserite nella loro ineluttabile storicità; la teoria dei numeri complessi è presentata come il settore in espansione della nuova fisica, il «campo morfogenetico », popolarizzato dalla filosofia del «New Age», è esposto come una delle teorie standard della gravitazione quantistica. La programmazione non lineare è progressista e libertaria contrariamente a quella lineare ed alla teoria dei giochi ${ }^{44}$. Per dirla in breve, in ogni paragrafo di questo scritto vi sono affermazioni assurde, riferimenti truffaldini, stravolgimenti pretestuosi di teorie, di dottrine, di dati storici.

Scritto in uno stile contorto, con frasi intere indecifrabili o incomprensibili, quest'articolo è da un capo all'altro così inintelligibile che la sua plausibilità dà immediatamente adito a sospetti, diffidenze e riserve.

Pochi giorni dopo la pubblicazione del fascicolo speciale della rivista « Social Text» sulla «Guerra delle scienze», Sokal avverte i redattori che il suo articolo è un pastiche, una parodia costituita da pezzi di più autori, di teorie inventate, di dottrine caricaturate, di tesi strambe, e propone di far uscire un breve testo rettificativo in questo senso. I redattori rifiutano e Sokal invia la precisazione ad una pic-

44 Per un'eccellente informazione su questi argomenti si rimanda a Giorgi, G. \& Guerraggio, A., Ha solo cinquanta anni: la programmazione non lineare, in PRISTEM/Storia. Note di matematica, Storia, Cultura, a cura di Pietro Nastasi, Milano, Springer, 1998, $\mathrm{n}^{\circ}$ 1, pp. 1-32 ed ivi anche l'art.di Dell'Aglio, L., Alcune questioni sull' evoluzione della teoria dei giochi, pp. 33-51. 
cola rivista universitaria, non molto diffusa, che la inserisce in un fascicolo già in corso di stampa. È la rivista, con un titolo emblematico, «Lingua franca».

In seguito ad un articolo uscito in un quotidiano locale, la stampa nazionale si precipita sull'avvenimento. La «beffa» è propagandata non solo dai quotidiani, dai settimanali, ma anche dalle televisioni.

L'ira e le proteste dei partigiani degli studi culturali sono durissime e scomposte. Sokal è accusato di disonestà, d'avere infranto le regole elementari della deontologia accademica, d'essere un plagiatore ed un impostore sfrontato. L'immoralità del procedimento è messo alla gogna, l'arroganza dello scientifico biasimata, il disprezzo per la cultura e per le scienze umane stigmatizzato. La parodia è denunciata come un inganno politico, un delitto di tradimento contro la Sinistra. I fascicoli di «Lingua franca», di «Dissent» e di «Tikkun» ospitano, tra luglio e dicembre del 1996, le polemiche e gli scontri tra Sokal ed i principali redattori di «Social Text», rivista ormai interdetta alla prosa sokaliana ${ }^{45}$. I siti su «Internet» consacrati all'avvenimento si moltiplicano in maniera esponenziale (finora 84.490), i forum di discussione (finora 560) vi sono frequentatissimi, l'amplificazione degli accadimenti è accelerata dalle onde della globalizzazione e della mondializzazione.

I difensori di Sokal tentano di riportare al centro della discussione il fatto che gli scienziati non mirano a svilire le scienze dell'uomo e della società, che essi vogliono solo correggere certi traviamenti intellettuali, ricordare che il mondo esterno esiste indipendentemente dalle idee che formuliamo su di lui, che le teorie scientifiche sono confermate o infirmate dalla realtà di questo mondo naturale preesistente agli osservatori, che la scienza produce conoscenze irriducibili a questo o quel contesto culturale e storico, che la ragione è lo strumento indispensabile per schiarire i misteri dell'Universo. In più, Sokal ed i suoi difensori ripetono a iosa di far propria la tradizione filosofica dell'Illuminismo che stabilisce un rapporto stretto tra l'universalismo ed il razionalismo, tra la ragione scientifica e l'emancipazione dell'uomo, e appunto per queste ragioni essi criticano il relativismo, il differenzialismo, la politicizzazione ad oltranza delle analisi scientifiche, la confusione tra la scienza, le sue applicazioni pratiche, le implicazioni socio-politiche e le conseguenze perverse di queste implicazioni, sia pure indirette, della stessa scienza.

Nessuno di questi argomenti è ripreso dagli avversari di Sokal. Essi persistono però, con una pertinacia davvero impressionante, a denunciare l'immoralità dell'impostura, gli attacchi sornioni alle scienze umane, la paura degli scienziati d'essere privati, ad opera degli storici, dei sociologici e dei filosofi, del monopolio degli studi sulle discipline scientifiche. Si rimprovera a Sokal d'avere abusato della fiducia dei colleghi e d'avere così intaccato la credibilità delle attività uni-

45 L'articolo in cui Sokal tenta di render conto delle ragioni che l'hanno indotto a scrivere la parodia, Transgressing the Boundaries: An Afterword, è rifiutato da «Social Texte», ma è pubblicato in «Dissent», 43, 1996, n 4, pp. 93-99 ed in una versione differente in «Philosophy and Literature $, 20,1996, n^{\circ} 2$, pp. 338-346. Vedere anche la trascrizione del dibattito oragnizzato, il 30 ottobre 1996, dalla New York University, in «New Politcs», 6, 1997, n² 2, pp. 126-129. Un'ottima analisi di questi scontri intellettuali è fornita da Dubois, M., $L^{\prime}$ "Affaire Sokal»: études culturelles et sociologie relativiste des sciences, «Revue française de sociologie», XXXIX, 1998, n³, pp. 391-418 e da Feldman, J., "L’Affaire Sokal»: un épisode de la méconnaissance entre culture, in «L'Année sociologique», 49, 1999, n¹, pp. 245-270. 
versitarie. Gli si rinfaccia altresì di fare delle norme epistemologiche e metodologiche vigenti nella fisica delle particelle il modello assoluto per il lavoro di tutte le scienze, anche per quelle dell'uomo e della società. Molti scrivono addirittura che il marxista ortodosso Sokal, irritato dall'abbandono progressivo dell'universalismo e dei valori scientifici da parte dei movimenti sociali di sinistra, a ciò incitati dai radicalismi femministi ed ecologici, con quel suo intervento tenta di discreditare $\mathrm{i}$ «gauchistes » e di restaurare le dottrine progressiste tradizionali.

Il dibattito nord-americano stava estinguendosi per mancanza d'argomenti nuovi allorché gli universitari francesi, criticati rudemente da Sokal per le loro opzioni relativiste ed irrazionaliste, per loro scarse e scarne conoscenze nelle discipline scientifiche, rilanciano le discussioni in Francia. Quivi la controversia prende proporzioni e tonalità vivissime ma ben diverse da quelle americane ${ }^{46}$.

\section{PARODIA, IMPOSTURA, CARICATURA DELLE SCIENZE UMANE?}

Il venerdi 20 dicembre 1996, un giornalista, Nicolas Weil, pubblica, sulla prima pagina del più importante quotidiano parigino del pomeriggio, un articolo a proposito di «the Sokal's hoax», intitolato La mistificazione pedagogica del professore Sokal ${ }^{47}$, articolo che offre una rapida cronaca degli avvenimenti ed insiste sulle critiche rivolte a Jacques Lacan, a Julia Kristeva, a Jacques Derrida, a Gilles Deleuze, à Félix Guattari, a Jean Baudrillard, a Bruno Latour, a Michel Serres, a Jean-François Lyotard, a Luce Irigaray ed a Paul Virilio, alla loro dilettantesca erudizione scientifica, ai loro imbrogli culturali, alle loro acritiche adesioni alla filosofia di Friedrich Nietzsche e di Martin Heidegger, alla loro infedeltà alla cultura dei Lumi.

Agli inizi del nuovo anno, Denis Duclos, molto noto in Francia per le sue belle ricerche di sociologia dei rischi industriali e della violenza, interviene nel dibattito per dire che le controversie sull' «affare Sokal» non appartengono alla vita intellettuale giacché non hanno come scopo la comprensione delle questioni in lizza, bensì quello di parodiare, denigrare, intrappolare, macchiare moralmente gli intellettuali francesi. L'«affare Sokal» è, per Duclos, un'operazione commerciale, una guerra psicologica condotta da un «pistoleros dell'intellectual correctness», uno sciovinismo anti-europeo, una manifestazione dell'imperialismo culturale americano, che non sopporta che la gioventù nord-americana ascolti i canti delle sirene europee ${ }^{48}$.

Il fisico belga Jean Bricmont tenta inutilmente di rettificare simili asserzioni, di riportare il dibattito al suo vero soggetto e di spiegare che l'unico obiettivo di Sokal è d'attirare l'attenzione degli addetti ai lavori sulla mancanza di serietà e di rigore nell'uso che certi studiosi francesi di scienze umane fanno della fisica e

46 Un'eccellente analisi delle ripercussioni in Francia è stata fatta da Mulligan, K., The Symptoms of Gödel-mania. Parisian abuse of science and postmodernist discourse, «The Times Literary Supplement », 1 May 1998, n 4961 . E dello stesso anche Valeurs et normes cognitives, «Magazine littéraire», n 361, janvier 1998, pp. 78-79.

47 Weil, N., La mystification pédagogique du professeur Sokal, «Le Monde», 20 dicembre 1996.

48 Duclos, D., Sokal n'est pas Socrate, «Le Monde», 3 janvier 1997. 
della matematica ${ }^{49}$. Lo stesso giorno e nelle stesse colonne Pierre Guerlain rammenta che le questioni sollevate da Sokal sono importanti; sostiene che vale la pena stabilire se veramente i giochi di parole abbiano preso il posto della riflessione, se i problemi sociali siano stati ridotti alla loro espressione semantica, se i post-modernisti riducano effettivamente la verità ad un rapporto di forze, ad un gioco di potere in un tempo ed in un luogo dati. E la sua conclusione è piena di buon senso: «Sokal n'a pas forcement raison contre les penseurs qu'il critique. Il a droit au respect et surtout les attaques ad hominem ne sauraient remplacer la discussion de ses thèses. Son canular ne fait que médiatiser une discussion qui a déjà eu lieu et qui n'est pas que lutte du scientisme contre les sciences humaines $»^{50}$.

L'invito alla discussione serena non è affatto accolto da colui che Sokal ha più rudemente bistrattato, Bruno Latour. Il suo intervento, sussiegoso, sprezzante, storna abilmente la discussione dall'epistemologia alla politica della scienza ${ }^{51}$. La reazione «scomposta» di Sokal rivelerebbe i timori di certi fisici teorici che non potendo più contare sulle sovvenzioni di cui disponevano durante gli anni della guerra fredda, devono oggi inventarsi dei nuovi nemici contro cui battersi per ottenere dei pingui finanziamenti. La scelta è caduta sugli intellettuali postmoderni venuti dall'Estero, particolarmente sui Francesi e su tutti quelli europei, asiatici ed africani che non ammettono l'egemonia culturale americana. «Nous assistons aux derniers soubresauts d'une science de guerre froide, mobilisée contre la religion, contre les Rouges, contre l'irrationalisme des masses.»

Le tesi dei sokalisti sarebbero una manifestazione di passeismo, una difesa d'interessi di bottega da parte di certi ambienti accademici, timorosi dei rischi insiti nel rapporto esistente tra la scienza e la politica, incapaci di riconoscere che la forza della scienza non è data dall'esattezza assoluta del suo sapere ma dalla qualità dell'esperienza collettiva che riesce a costruire insieme con altri, dal contributo che sa dare alla composizione progressiva d'una volontà comune di resistere al destino. Gli attacchi agli studi sociali delle scienze sarebbero i sintomi del rifiuto d'analizzare realisticamente le scienze, di riconoscere i legami che stringono i ricercatori, gli strumenti, i laboratori, le pratiche, i concetti, gli oggetti scientifici, i testi prodotti dagli scientifici, colla cultura, colla storia, colla vita pubblica, con il cosmo. «Comment pourrait-on voir des ennemis à abattre dans ces chercheurs attentifs au monde de la recherche, à son histoire, à ses crises? Il faut se faire aux autres réalités de la vie: les faits ne naissent pas des choux!»

Sokal, pazientemente, precisa che le tesi contro il relativismo post-moderno sono argomentate da sociologi, storici, filosofi, letterati, e non dai soli fisici. In più, nessuno ha mai avuto l'intenzione di limitare il credito di cui gli universatari francesi godono sui campus americani. Quelle tesi hanno un solo ed unico obiettivo: difendere la sinistra universitaria americana contro le tendenze irrazionaliste alla moda, tendenze che rischiano di metterla in pericolo, anzi di condurla al suicidio. Infine, che Latour dismetta il tono saccente ed altezzoso, finisca di mescolare allegramente ontologia ed epistemologia, di svuotare la scienza dei suoi contenuti cognitivi, di riempirla di trivialità, come per esempio quella che fa della

\footnotetext{
49 Bricmont, J., La vraie signification de l'affaire Sokal, «Le Monde», 14 janvier 1997.

50 Guerlain, P., Haro français sur le professeur américain, «Le Monde», 14 janvier 1997.

51 Latour, B., Y a-t-il une science après la guerre froide?, «Le Monde», 18 janvier 1997.
} 
teoria einsteiniana della relatività «un contributo alla sociologia della delega », e ci dimostri infine in che modo si possa arrivare a spiegare la storia delle scienze senza tener conto della verità o della falsità delle teorie, senza mai ricorrere alle prove empiriche in favore o a svantaggio d'esse. Nella situazione attuale, conclude Sokal, tutti dovrebbero far prova d'un maggiore rigore intellettuale. «Le scienze esatte e le scienze 'molli' sono a bordo dello stesso battello. Flirtare col relativismo e l'irrazionalismo non le condurrà in nessun porto. ${ }^{52}$

Jean-Jacques Salomon, Michel Rio, Régis Debray ${ }^{53}$, tra gli altri, difendono, con sfumature diverse, la battaglia contro il relativismo, contro la riduzione del discorso scientifico ad un equivalente del mito o d'un poema surrealista, mentre Sally Wyatt, Hervé Le Bras, Isabelle Stengers et Jean-Marc Lévy-Leblond ${ }^{54}$, insieme con numerosi altri studiosi, rivendicano il diritto di conoscere quali sono gli orientamenti della ricerca scientifica, come le scienze e le ideologie s'inseriscono nella società, d'apprezzare positivamente la validità del programma di ricerca degli studi sociali delle scienze. Per questi studiosi la querelle sokalista è una farsa volgare, un marchingegno per ridicolizzare la necessità assoluta (a detta di nuovo del Latour) di rivelare le poste politiche in gioco nei dibattiti scientifici, di rovesciare l'intersoggettività in interoggettività, di cambiare le nozioni d'attore e d'azione grazie alla presa in considerazione degli agenti umani e non umani, allo stabilimento per tutti gli oggetti, nell'ambito d'un sistema d'interazioni collocate in un proprio contesto, dello statuto d'attori sociali a pieno titolo ${ }^{55}$.

Nel mese di settembre 1997 Alain Sokal e Jean Bricmont fanno uscire a Parigi il libro Impostures intellectuelles, nel quale riuniscono un florilegio di citazioni e commentano scritti di rinomati autori molto alla moda nei mass media e che rivelano le mistificazioni fisico-matematiche, la speciosa erudizione di queste celebrità molto in voga anche nelle università, nelle riviste culturali e nei periodici in vista degli Stati Uniti d'America.

I quotidiani, le radio, le televisioni della francofonia approfittano della pubblicazione per rilanciare il dibattito e per dare la parola a tutti quelli bistrattati dai due fisici nonché a coloro che recepiscono il libro come un attacco all'intera cultura francese o come una stroncatura inaccettabile delle scienze dell'uomo e della società.

52 Sokal, A., Pourquoi j' ai écrit ma parodie, «Le Monde», 31 janvier 1997. Nell'articolo si fa allusione allo scritto di Latour, B., A Relativist Account of Einstein's Relativity, «Social Studies of Science», 18, 1988, pp. 3-44.

53 Salomon, J.-J., L'éclat de rire de Sokal, «Le Monde», 31 janvier 1997; Rio, M., Grâce au ciel, à Sokal et à ses pareils, «Le Monde», 11 février 1997; Debray, R., Savants contre docteurs, «Le Monde », 18 mars 1997.

54 Wyatt, S., Sociologie de la connaissance scientifique, «Le Courrier», 20 février 1997; Le Bras, H., Une farce sans valeur d' enseignement, «La Recherche», n²99, juin 1997; Lévy-Leblond, J.M., La paille des philosophes et la poutre des physiciens, «La Recherche», $\mathrm{n}^{\circ} 299$, juin 1997; Stengers, I., Inventer une écologie des pratiques, «La Recherche», n² 297, avrile 1997.

55 Latour, B., Un nouveau délit d'opinion: faire de la politique, «Le Monde», 4 octobre 1996; Esquisse d'un parlement des choses, «Écologie politique», n 10, Été 1994, pp. 97-115; Une sociologie sans objet? Remarques sur l'interobjectivité, «Sociologie du travail», 1994, n 4, pp. 587-607; Moderniser ou écologiser? À la recherche de la «septième» cité, «Écologie politique», $\mathrm{n}^{\circ} 13,1995$, pp. 5-27. 
Due fisici tentano di mettere un po' d'ordine in tanti sciamannati interventi, di far ragionare i piagnoni, di ridimensionare la portata delle critiche di Sokal e Bricmont ${ }^{56}$. Anche costoro tentano di precisare con nettezza i loro intenti, dichiarano che non disprezzano le scienze umane, che denunciano soltanto gli usi indebiti che queste scienze fanno delle nozioni fisiche e matematiche e che la loro principali preoccupazioni restano le questioni del relativismo, dell'universalismo, della verità, dell'oggettività, della validità delle proposizioni scientifiche ${ }^{57}$. Queste dichiarazioni non producono nessun effetto.

Verso la fine del mese di novembre del 1997 interviene nella discussione il filosofo Jacques Derrida e con tono altero dichiara che le polemiche suscitate da Sokal non meritano alcuna considerazione. Il fisico americano ha come scopo di discreditare i professori europei che insegnano negli Stati Uniti e che secondo certi intellettuali americani hanno un prestigio esorbitante ed ingombrante. La campagna che costoro hanno montato manca di probità, di rettitudine, di serietà, di onestà. "Qui avait intérêt à se précipiter sur une farce au lieu de participer au travail dont elle a tristement tenu lieu? Engagé depuis longtemps, ce travail se poursuivra ailleurs et autrement, je l'espère, de façon digne: à la hauteur des enjeux.» $^{58}$

A Derrida fa subito eco il professore di psichiatria Max Dorra per ricordare a tutti che l'utilizzazione metaforica dei concetti scientifici risale a Eraclito ed a Platone, che i modelli scientifici non disdegnano l'assistenza delle metafore (la doppia elica, l'ADN, ecc.), che le metafore facilitano, a certe condizioni, l'elaborazione di ipotesi interpretative e permettono d'affinare, precisare la natura d'un fenomeno in corso di studio. Le metafore sono le vestigia d'una soggettività che il feticismo scientista vorrebbe cancellare, come vorrebbe occultare i conflitti e la politica per poi far credere che la realtà è neutrale. Purtroppo le stroncature di Sokal concernono autori che analizzano il potere, «ce pouvoir qui se résume si souvent, en fin de compte, à la capacité d'angoisser». Appunto per questo «Il ne faut pas se tromper d'imposteurs. ${ }^{59}$

I chiamati in causa si limitano a constatare che gli intervenuti nel dibattito non formulano critiche precise. Tutti ammettono implicitamente che ciò che è stato detto è veritiero ma che, per diversi motivi, non bisognava dirlo. Per quanto riguarda le metafore, Sokal e Bricmont ripetono di non essere contrari al loro impiego. Ciò che invece contestano e che i denigratori fanno finta d'ignorare, è l'uso di concetti tecnici al di fuori dei contesti d'origine e senza giustificazione della loro pertinenza. Si cita a titolo d'esempio: «insieme compatto», «funzione discontinua », «gruppoidi, monoidi», «strutture di corpo e di campo», «iperspazio a rifrazione multipla», «spazio dromosferico», «compacità topologica»e così di seguito ${ }^{60}$.

\footnotetext{
56 Treiner, J., Sokal-Bricmont: non, ce n'est pas la guerre, «Le Monde», 11 octobre 1997; Krivine, H., Quel impérialisme?, «Le Monde», 11 octobre 1997.

57 Bricmont, J. \& Sokal, A., Que se passe-t-il?, «Libération», 18 et 19 octobre 1997, pp.5-6.

58 Derrida, J., Sokal et Bricmont ne sont pas sérieux, «Le Monde», 20 novembre 1997.

59 Dorra, M., Métaphore et politique, «Le Monde», 20 novembre 1997.

60 Bricmont, J. \& Sokal, A., Réponse à Jacques Derrida et Max Dorra, « Le Monde», 12 décembre 1997.
} 
La cronistoria della polemica mostra molto bene che diversi argomenti sono stati dibattuti (l'imperialismo culturale americano, l'egemonia delle scienze dure, l'unicità e/o la pluralità dei modelli cognitivi, le metafore e le analogie, le relazioni della scienza colla politica) ma che né Sokal-Bricmont né gli intervenuti hanno rigorosamente e nettamente distinto il relativismo, che riduce la scienza ad un testo o a un prodotto delle sole condizioni socio-storiche, dall'uso dei termini scientifici al di fuori dei contesti originari. Sarebbe stato necessario dare più precisioni sul relativismo.

Esiste un relativismo filosofico, un relativismo culturale, un relativismo etnologico, un relativismo antrolopologico, un relativismo metodologico, un relativismo axiologico e tante e diverse pratiche relativistiche seguite dagli storici e dai sociologi, pratiche che sarebbe stato opportuno, per la chiarezza del dibattito, esplicitare. Rifiutare di gerarchizzare e comprendere non implicano l'equivalenza generalizzata di tutte le cose né che esse debbano essere necessariamente approvate o accettate. Perciò la distinzione tra il relativismo culturale dell'etnologo ed il relativismo axiologico del filosofo appare a tutti insormontabile ${ }^{61}$.

Per quanto riguarda l'abuso che certi studiosi di scienze umane fanno dei termini scientifici, il problema non è così grave che si possa immaginare. Un concetto può ricevere un altro senso se situato in un altro contesto. L'analisi rende chiaro e spiega questo uso e ciò non ha mai costituito un delitto di lesa epistemologia della scienza. In un certo senso, ha ragione Henri-Pierre Jeudy di dire che abbiamo assistito ad un pugilato epistemologico anziché ad una vera controversia scientifica e di ricordare agli addetti ai lavori che l'intervento massiccio dei mass media ha traviato la «querelle» e le ha dato un contenuto ed una finalità che all'origine non esistevano: «Une aventure de la pensée si bien médiatisée finit par être institutionnalisée même si elle est rejetée par les partisans d'une quelconque orthodoxie scientifique. Imposant des modèles de langage et d'interprétation des phénomènes de société et du monde, elle s'exténue au rythme des effets de mode qu'elle ne cesse de renouveler. Entre le snobisme intellectuel et le néo-scientisme, n'y a-t-il plus d'issue? Sans doute est-il préférable d'en référer à l'écriture ellemême en reconnaissant que seule la souveraineté du style est en mesure de donner à l'aventure des idées sa puissance de fiction. ${ }^{62}$

Un'analisi del contenuto di tutti gli scritti usciti in Francia sull' «affare Sokal» rivela, senza tema di smentita, che il giudizio espresso è globalmente negativo benché le motivazioni che lo hanno determinato compongono una gamma di gradazioni eterogenee. Gli eccessi, ovviamente, sono, in questo genere di cose, ineluttabili. La palma della stravaganza potrebbe essere attribuita a quelli che si sono arrabattati a stabilire accostamenti tra il fisico A. Sokal ed il procuratore speciale K. Starr, come per esempio quel ben noto filosofo parigino, titolare d'una rubrica settimanale in un grande e rispettabile giornale, il quale non ha esitato a scrivere che il parallelismo tra l'inquisitore del presidente Clinton ed il professore di fisica ci rivela i legami esistenti tra l'ordine scientifico e l'ordine morale e ci mostra le due facce dello stesso puritanismo rigorista, le due maniere più inte-

61 Ascher, E., Problèmes du relativisme, «Revue européenne des sciences sociales », XXVII, 1989, $\mathrm{n}^{\circ} 83$, pp. 123-146.

62 Jeudy, H.-P., Requiem pour un moralisme scientifique, «Le Monde», 12 décembre 1997. 
griste di coltivare l'odio, di squalificare i vicini e di trasformali in nemici acerrimi $^{63}$.

Gli echi delle discussioni s'erano appena spenti allorché Yves Jeanneret, professore di scienze dell'informazione e della comunicazione nell'Università di Lilla-III, propone un bilancio consuntivo ed un inventario quantitativo degli articoli, degli interventi radiofonici e televisivi, insomma di tutto ciò che è stato scritto e detto a proposito dell' «affare Sokal» in Francia. Da tanto lavoro l'autore ne cava un poco irenico giudizio sintetico ${ }^{64}$.

Gli scritti di Sokal, sottomessi ad una radioscopia minuziosa persino dei titoli, dello stile, della costruzione delle frasi, gli appaiono ben povera cosa. Vi si parla di fatti e non vi sono che dei testi; vi si chiama in aiuto il reale e vi si osserva il trionfo della finzione; vi si discorre di costanti e non vi si vedono se non delle variabili; vi si celebra la scienza e vi domina la letteratura; vi si invocano i Lumi ed è lo spettro dell'impostura, della contraffazione, dell'estrapolazione a signoreggiarvi. In quegli scritti al posto della Ragione Sokal ha messo in trono un suo mostruoso simulacro.

Il giudizio è malevolo, astioso, tendenzioso. Le critiche sono eccessive e talvolta anche speciose. Un vero e proprio processo è intentato a ciò che si presume che Sokal abbia voluto veramente fare. Le critiche degli avversari sono prese in considerazione mentre la posizione del processato è negativamente data per scontata. La sentenza è ancora più bislacca: «Lu dans sa globalité, le texte de la querelle exprime essentiellement un exorcisme trivial. [...]. Ce discours prétend que l'échange n'a pas lieu d'être, que l'influence intellectuelle est néfaste, que les bons savoirs sont ceux qui se referment sur eux-mêmes.»

Agente della cultura della trivialità, il fisico Sokal sarebbe all'origine d'una impostura dissimulata. «Elle qui fait prendre la vessie d'une manœuvre éditoriale pour la lanterne de l'expérimentation, elle précipite la condamnation d'auteurs non lus qui entraînera inéluctablement la mise au pilori des autres; elle fait prendre pour agent comptant le fait qu'il y ait des post-modernes et un projet des Lumières. ${ }^{65}$

La raccolta di scritti ${ }^{66}$ curata da un altro professore di scienze dell'informazione e della comunicazione nell'Università di Parigi-VII, Baudouin Jurdant, è certo meno tendenziosa del libro di Jeanneret. Né l'introduzione né i sedici articoli di questa raccolta fanno prova d'una compassionevole equanimità, ma nell'insieme l'informazione è affidabile, la polemica ha toni accettabili, e gli argomenti contro Sokal suscettibili d'essere discussi pacatamente. Divisa in quattro parti, la raccolta tenta di rispondere alla domanda « Chi approfitta della buona fede di chi?», e poi d'indicare in che maniera bisogna parlare delle scienze, se

63 Droit, R.-P., Nous sommes tous des imposteurs!, «Le Monde», 2 octobre 1998.

64 Jeanneret, Y., Décontamination intellectuelle : l'expérimentation échoue, «L'aventure humaine», n 8 , décembre 1997, pp. 81-89, e poi in maniera più che dettagliata nel libro L'affaire Sokal ou la querelle des impostures, Paris, PUF, 1998.

65 Jeanneret, Y., L'affaire Sokal..., op. cit., p. 251 e p. 253.

66 Sous la direction de Jurdant, B., Impostures scientifiques. Les malentendus de l'affaire Sokal, op. cit. e $2^{\mathrm{a}}$ ed. corretta, ibidem, 1999. Una raccolta d'articoli sull' «affare Sokal», fatta con criteri imparziali ed una lodevole apertura di spirito, è stata pubblicata dalla rivista fondata da Jean-Paul Sartre, «Temps modernes », nº 600, juillet-août-septembre 1998. 
Sokal e Bricmont hanno letto e capito gli scrittori che criticano e se esistano le condizioni per colmare il fossato esistente tra le scienze dure e le scienze molli.

Lasciando da parte gli sviluppi sulle modulazioni morali, sulle implicazioni politiche, sull'ostilità positivista nei riguardi delle scienze umane, sul trattamento « iniquo» riservato agli studiosi francesi, molte osservazioni contenute nei sedici articoli sono sensate, e quindi condivisibili. Ciò che Lévy-Leblond ${ }^{67}$ scrive a proposito delle metafore, della confusione tra costruttivismo e relativismo, sui punti che la validazione d'un enunciato non dice nulla sul suo significato e sulla sua pertinenza, che le scienze umane vanno valutate in base ai criteri interni a queste scienze e non già in base alle epistemologie delle scienze sperimentali e delle scienze formali, - tutto ciò è accettabile e costituisce un buon contributo al dibattito scientifico, come sembra saggio ed opportuno ricordare che nelle comunità intellettuali i malintesi sono inevitabili, per cui «La fécondité des échanges demande plus d'attention mutuelle que de jugements péremptoires, plus d'écoute modeste que de condamnations sans appel.»

Per quanto riguarda l'universalismo ed il relativismo e la specificità delle scienze dell'uomo e della società, i sedici contributi restano tutti in bilico tra l'ambiguità e l'esitazione. A questo proposito, il solo contributo che pone dei problemi suscettibili d'una discussione costruttiva è quello di due storici delle scienze, che ricordano non soltanto quanto sia necessario precisare di quali forme di relativismo s'intende discutere ma anche ch'è impossibile contestare l'esistenza del mondo reale. Il problema da risolvere è quello di stabilire in che mondo possiamo rendere conto di questo mondo e secondo quali criteri dobbiamo giudicare l'adeguatezza delle nostre costruzioni teoriche su di esso. Le scienze sono efficaci perché aiutano l'azione degli uomini, perché sono azione utile, pratica, perché ci permettono d'agire sul mondo materiale, di modificarlo, di controllarlo ${ }^{68}$.

Si può essere senz'altro d'accordo cogli autori che le scienze nascono nell'azione e per l'azione, ma non è storicamente dimostrabile che tutti i processi cognitivi siano sempre riducibili a questa unica ragion d'essere.

Non sono molto convincenti i quattro articoli ${ }^{69}$ in cui si dimostra che Sokal e Bricmont non hanno letto gli autori da loro criticati. I due fisici non avevano certo l'intenzione di propore una lettura filologicamente fedele e nemmeno darcene un'interpretazione rispettosa delle regole dell'ermeneutica. Si sono limitati a ricercare, con il metodo della campionatura, nei testi, scelti casualmente, concetti ed espressioni, mutuati alla fisica ed alla matematica, ed il cui uso, a loro avviso, è improprio o scorretto. Certo, i due sono persuasi, a torto, che questi concetti e queste espressioni ricevono il solo ed unico senso esclusivamente non dal contesto in cui sono ora inseriti ma da quello d'origine. Tale menda è grave ma

67 Lévy-Leblond, J.-M., La méprise et le mépris, in Impostures scientifiques..., op. cit., pp. 27-42.

Dahan Delmedico, A. \& Pestre, D., Comment parler des sciences aujourd'hui?, in Impostures scientifiques..., op. cit., pp. 77-105.

69 Redatti nell'op. cit.da J.-M. Salanskis (Pour une épistémologie de la lecture, pp.157-194), da N. D. Mermin (Physiciens, encore un effort!, pp. 195-201), da D. Fisari (Sokal lisant Latour lisant Einstein : rire sans lire ou lire sans rire?, pp. 202-213), da J. H. Fujimura (L'autorité du savoir en question, pp. 214-236), da N. Charraud (Mathématiques avec Lacan, pp.237-249). Su questi saggi ved. le osservazioni di Dubois, M., Impostures et malentendus, in «Revue européenne des sciences sociales », XXXVII, 1999, nº 115, pp. 267-271. 
non tanto da autorizzarci ad accusare i due autori di non aver fatto quello che essi non volevano e non dovevano fare.

La quarta parte del volume è un'apologia, né nuova né originale, pro domo, degli studi sociali delle scienze, scritta da un studioso in prima linea in questo settore, Michel Callon ${ }^{70}$, il quale rigetta l'accusa d'irrazionalismo e ridimensiona quella di relativismo. L'articolo di Isabelle Stengers ${ }^{71}$, di tono irenico e di portata ecumenica, riprone il problema classico dei rapporti tra la scienza e le umanità nonché la questione dei malintesi esistenti tra i fisici, i filosofi, gli storici ed i sociologi. La soluzione proposta, rifiutare le parole d'ordine tradizionali e le interpretazioni normative standard, resistere con tutti i mezzi, e su tale rifiuto fondare un modello cognitivo nuovo, è molto irrealista. Essa non tiene conto delle tradizioni disciplinari, delle specificità delle istituzioni in cui le discipline sono inserite e di tutte le particolarità storiche. Molto seducente è la frase che dice «Lorsque sont visés des groupes humains, ces moyens sont ce que nous appelons la capacité sociale et politique de s'autodéfinir. Les sciences humaines et sociales devraient donc être aussi rares et dépendantes de l'événement que les sciences expérimentales. Lorsqu'il s'agit des humains, nous ne pouvons espérer de connaissance fiable que là où, historiquement et politiquement, nous la méritons.». Purtroppo le capacità sociali e politiche d'autodefinazione dei gruppi umani sono ancora un mistero e non si sa bene quale potrebbe essere l'oggetto delle scienze umane se esse potessero realmente affrancarsi dagli accadimenti e dalla «histoire événementielle».

Questo articolo e tanti altri contenuti nel volume curato da Jurdant sono preziosi perché ci dimostrano come una controversia su un tema preciso possa essere stravolta e deviata dal suo corso se pubblicizzata dai mass media. Allain Glykos ha ragione di concludere il volume colla constatazione che la scienza soggiace al diktat della finanza e dei mass media e che grazie alla mondializzazione dei processi di produzione e di diffusione «la montée de certaines idéologies extrémistes peuvent raviver le feu, même là où certains pensent qu'il est déjà éteint $»^{72}$.

La lezione che si può cavare da questa controversia è semplice: né gli equivoci del relativismo, né le affinità tra il relativismo cognitivo e l'ideologia differenzialista, né i modelli di spiegazione proposti dagli studi sociali delle scienze, né la questione dell'unità epistemologica e metodologica delle scienze o del loro pluralismo, sono stati affrontati. Quanto tempo ancora bisognerà aspettare in vista d'un chiarimento di cui ormai abbiamo un urgente bisogno ${ }^{73}$

70 Callon, M., Défense et illustration des recherches sur les sciences, in Impostures scientifiques..., op. cit., pp. 253-267.

71 Stengers, I., La guerre des sciences: et la paix?, in Impostures scientifiques..., op. cit., pp.268292. Della stessa ved.anche Sciences et pouvoirs. Faut-il en avoir peur?, Bruxelles, Ed. Labor, 1997.

72 Glykos, A., Une affaire peut en cacher une autre, in Impostures scientifiques..., op. cit., pp. 293313.

73 Per degli ulteriori sviluppi rimandiamo a Busino, G., Combats pour la sociologie, Lausanne, IASUL, 1998. 


\section{CORTO INTERMEZZO ITALIANO}

Quali sono state le ripercussioni in Italia di questi sconvolgimenti intellettuali nello studio delle scienze e della controversia scatenata dagli scritti di Sokal e poi dal libro di Sokal et Bricmont?

I filosofi della scienza che hanno contribuito a riformulare le problematiche delle scienze (Y.Elkana, N. R. Hanson, T. S. Kuhn, I. Lakatos, A. Musgrave, ed ovviamente K. Popper e tanti altri) sono stati largamente tradotti e commentati nella penisola. Le discussioni sociologiche vi sono state accuratamente presentate $^{74}$. Recentemente il saggio La dimensione sociale della conoscenza di David Bloor è stato tradotto in italiano con un'introduzione d'Alessandro Dal Lago ${ }^{75}$. Il libro di Gabriele Lolli, da poco pubblicato, fornisce succintamente una ragionata illustrazione delle peripezie che il dibattito tra i realisti ed i relativisti ha conosciuto dai tempi di Wittgestein a quelli di Sokal ${ }^{76}$.

Armando Massarenti, a metà giugno del 1997, presenta una rapida cronaca della «beffa Sokal», ne sottolinea il carattere divertente, beffardo, anti-ideologico, ma anche la sua «utilità pubblica» giacché pone il problema della valutazione dell'attività scientifica, dei rapporti scienza-cultura, scienza-società e anche di «quanto controproducenti, socialmente e culturalmente» possano «diventare il relativismo e il soggettivismo impliciti nella prassi e nei dogmi degli amici progressisti ${ }^{77}$. La replica di Gianni Vattimo è stizzosa. Sospetta delle manovre contro la filosofia di impianto heidegerriano elaborata ai giorni nostri e forse perciò qualifica lo scritto di Sokal «un saggio pieno di assurdità nascoste sotto un complicato linguaggio derrediano-lacaniano», uno scherzo che lascia il tempo che trova ${ }^{78}$. Massarenti ritorna sull'argomento ma la discussione non ha più per oggetto Sokal bensì il valore cognitivo della logica ed il suo ruolo nella ricerca filosofica ${ }^{79}$.

Ulderico Urzi scriverà che gli Americani attaccano gli intellettuali parigini definiti impostori, falsari, pasticcioni, truffatori in numerose interpretazioni del pensiero scientifico, ma che i Francesi considerano ormai il docente di fisica Alan Sokal «alla stregua d'un 'chierico traditore' da portare sulla ghigliottina ${ }^{80}$. Massarenti stigmatizza le reazioni scomposte, fatte d'insulti, di sciovismo culturale, e nello stesso tempo constata lo scarso interesse che la polemica ha suscitato in Italia. Molto opportunamente e saggiamente nota che le critiche di Sokal e Bricmont non sono «né un giudizio globale su una Nazione, né sull'opera complessiva degli

74 Ricordiamo soltanto la rassegna di Agodi, M. C., La sociologia della conoscenza ed il problema dei fondamenti delle scienze. La debolezza del programma «forte», «Sociologia», N.S., XX, gennaio-aprile 1986, pp. 85-171, con un'ampia bibliografia, ed il saggio di Cassano, F., Il gioco della scienza, «Rassegna italiana di sociologia », XXX, n 1, gennaio-marzo 1989, pp. 3 -30.

75 Milano, Raffaello Cortina, 1994.

76 Lolli, G., Beffe, scienziati e relativismo, op. cit.

77 Massarenti, A., L' utilità pubblica della beffa Sokal, «Il Sole-24 ore», 15 giugno 1997.

78 Vattimo, G., Troppo buon senso anti-Severino, «La Stampa», 16 giugno 1997.

79 Massarenti, A., Il buon senso non è mai troppo, «Il Sole-24 ore», 22 giugno 1997. Vedere anche di Severino, E., In difesa di don Benedetto il filosofo poco «logico», «Corriere della sera», $1^{\circ}$ novembre 1997.

80 Urzi, U., «Francesi, intellettuali impostori», «Corriere della sera», 26 settembre 1997. 
autori citati, né tantomeno di un attacco alla cultura umanistica in generale sferrato dai soliti scienziati arroganti. [...] Il libro di Sokal e Bricmont ha il merito di smascherare certi atteggiamenti della nostra cultura, certi tic, profondi e irriflessi, di stampo chiaramente conservatore, cui tutti siamo soggetti. Essi generano tra l'altro una serie di malintesi sul rapporto tra 'le due culture', quella umanistica e scientifica, e depotenziano in quest'ultima proprio 'il suo insegnamento metodologico più prezioso’: quello contrario al principio di autorità. E il sospetto è che quel nostra - senza voler incoraggiare un'indagine simile sulla filosofia italiana, che risulterebbe ridondante - non riguardi solo francesi e americani $»^{81}$.

Anche Pier Aldo Rovatti giudica positivamente l'operazione Sokal e formula il voto che le scienze umane accettino di riflettere sulle problematiche da essa sollevate e suscitate ${ }^{82}$. Da parte sua Roberto Festa dà la parola a Sokal, che spiega ancora una volta le ragioni dei suoi interventi, nota che nessuno ha risposto alle questioni da lui poste, che i decostruzionisti si limitano a giudicarlo un malato di mente, un agente della politica economica e dell'egemonia degli Stati Uniti, che non ha voluto in nessun momento fissare regole, prescrizioni, indirizzi di ricerca per le scienze dell'uomo e della società. Una sola preoccupazione l'ha spinto ad agire: far prendere coscienza che il decostruzionismo veicola un «relativismo culturale e cognitivo che tratta le scienze come delle narrazioni o delle semplici costruzioni sociali. Con Bricmont abbiamo inteso invece difendere la razionalità e la credibilità della sinistra intellettuale $»^{83}$.

I giornali ed i periodici italiani hanno riportato le cronache delle peripezie dell' «affare Sokal» senza troppo simpatia per le reazioni dei Francesi. Senonché il problema di fondo, quello che costituisce la sola cosa importante ed interessante di tutta questa vicenda, cioè la natura del rapporto tra universalismo e relativismo, tra scienze dure e scienze molli, tra le scienze e la ricerca, tra la cultura e la società, - questo problema di fondo non è stato purtroppo affrontato come sarebbe stato invece opportunamente fare.

\section{IN GUISA DI CONCLUSIONE PROVVISORIA}

La prima osservazione da fare è che la discussione è stata deviata dal suo corso normale dall'intervento massiccio dei mass media, i quali hanno valorizzato solo i temi che potevano suscitare l'interesse dei lettori e degli spettatori. Le regole e le convenzioni di questi organi di diffusione tengono conto, ovviamente, dei pubblici destinatari di cui presuppongono le attese particolari. Le forme del discorso e gli stili di ragionamento sono contaminati da codesta presunzione, dalle limitazioni del tempo e dello spazio disponibili, dalla forma della comunicazione contraria alle distinzioni, alle sfumature, insofferente dei giochi linguistici tra il senso figurato ed il senso letterale.

\footnotetext{
81 Massarenti, A., Quei ciarlatani fra due culture, «Il Sole-24 ore», 19 ottobre 1997.

82 Rovatti, P. A., Che truffa quel pensiero francese, «La Repubblica», 21 dicembre 1997.

83 Festa, R., Sokal: «La scienza non è narrazione. Cari filosofi, maneggiatela con cura», «L'Unità », 3 dicembre 1997.
} 
Detto ciò, bisogna convenire che la questione dello statuto della scienza è importante e merita un'attenta considerazione. Possiamo distinguerlo da quelli dell'ideologia, della pseudo-scienza e della non-scienza? In che modo?

È indiscutibile che gli aspetti intellettuali della questione prima si mescolano e poi si confondono con esigenze ed attese istituzionali (valutare i lavori, acquisire e mantenere la reputazione, ottenere fondi per la ricerca, avere riconoscimenti accademici, ecc.), ma esistono effettivamente ragioni valide per non distinguerli? È legittimo fare una teoria causale delle conoscenze scientifiche che le confonda colle pratiche istituzionali cui queste conoscenze danno luogo? C'è da dubitarne. Sappiamo, invece, e con certezza, che la distinzione tra l'aspetto cognitivo/intellettuale e l'aspetto istituzionale ci aiuta a porre in maniera rigorosa il problema del relativismo che, almeno nelle sue formulazioni più radicali, mette in dubbio la possibilità stessa di stabilire una differenza tra la scienza e la non scienza.

Credo che Sokal abbia torto di non separare la posizione di coloro che abusano senza ragioni valide dei concetti scientifici da quella di coloro per cui la scienza è una costruzione sociale, un discorso che riposa su fattori a lei preesistenti. A torto però gli si rimprovera d'assolutizzare la scienza, di considerarla sacra. Al contrario, egli ne ammette i limiti pur rivendicandone la specificità e l'efficacia cognitive. Non contesta tutte le forme di relativismo ma soltanto le più radicali, quelle che affermano essere la scienza una forma di conoscenza avente lo stesso statuto della magia, dell'astrologia e della religione, che le teorie scientifiche sono costruzioni fondate su presupposti arbitrari, frutto di rapporti di forza sempre correlati ad interessi economici, sociali, politici e culturali, che non si fa scienza senza fare della politica, del diritto, dell'etica, della comunicazione, dell'economia benché in tutti questi campi i metodi di validazione siano disparati e sovente differenti. Appunto perciò Latour ha scritto: «The whole process of fact construction has been shown to be accountable inside a sociological framework.» ${ }^{84}$ Appunto perciò può dire che la scienza è stata sempre associata alle attività militaro-guerresche, che è sempre stata dotata di programmi basati sull'onniscienza e d'una volontà brutale di modernizzare ogni cosa. Appunto perciò predica e magnifica una scienza immersa nella cultura, nella politica, totalmente sprofondata nel mondo sociale.

Un'analisi dettagliata delle diverse forme di relativismo (la psicologica, la storica, la culturale, la fillosofica, l'axiologica, l'epistemologica), delle sue differenze con l'universalismo e con l'assoluto, avrebbe forse messo meglio in evidenza che le critiche di Sokal sono rivolte innanzitutto al relativismo assoluto, espresso dal quel principio di simmetria per il quale la spiegazione causale deve trattare nella stessa maniera la verità e la falsità, la razionalità e l'irrazionalità, l'ADN ed i fluidi umorali o la ghiondola pineale. Una tale precisazione avrebbe sottolineato che le teorie di Newton, di Darwin, di Einstein e di tanti altri sono state elaborate, effettivamente, in determinati contesti storici ma che esse contengono delle «verità ». E sono appunto queste «verità » a farle sopravvivere, in tutto o in parte, al di fuori dei contesti d'origine. Dei fatti oggettivi decidono, in ultima istanza, se le tesi, le

84 Latour, B., Is it possible to reconstruct the research process? Sociology of a brain peptide, «Sociology of the Science Yearbook», 4, 1980, pp. 43-76. La cit. si trova a p. 53. Sulla complessa questione del ricorso all'analogia disponiamo oggi dell'aureo libro di Bouveresse, J., Prodiges et vestiges de l'analogie. De l'abus des belles-lettres dans la pensée, Paris, Raison d'Agir éd., 1999. 
proposizioni, gli enunciati, formulati in contesti determinati, con un vocabolario dato, sono veri oppure falsi.

A ragione à stato notato che «La pesuasion rationnelle, [...], consiste en la dissémination de pratiques scientifiques au-delà du contexte local qui les a fait naître [...] La ratiocination et l'inférence jouent un rôle vital en disséminant les pratiques culturelles au-delà de leur contexte local, puisqu'elles servent à convaincre des communautés rivales que les pratiques en litiges sont représentatives de vues comprises dans leurs propres pratiques $»^{85}$.

La controversia non ha dato nessuna risposta alla domanda se il razionalismo occidentale sia un valore come tutti gli altri valori sociali, se il tentativo di Max Weber di descrivere la singolarità culturale, d'interpretarla come non universalità ma nello stesso tempo d'universalizzarla grazie col metodo storico-comparativo, se questo tentativo meriti d'essere sostenuto e perseguito, se abbia o possa avere una qualche validità, se valga la pena di distinguere la scienza dagli usi che se ne possono fare in tempi e contesti socio-culturali diversi ${ }^{86}$.

L'incomprensione totale che questo dibattito ha messo in evidenza deve insegnarci a non mescolare le questioni epistemologiche con quelle etiche, le questioni di fatto con le questioni di valore, le questioni cognitive con quelle sul funzionamento della scienza, a domandarci se la spiegazione e la comprensione possano provenire dallo stesso modello cognitivo. Se spiegare vuol dire imputare un accadimento ad una causa o ad una legge, l'attribuzione del senso appartiene allora ad un altro ordine. Il che solleva la questione della specificità delle scienze dell'uomo e della società rispetto alle scienze formali e sperimentali.

Purtroppo tali questioni non sono state esaminate dai partecipanti alla controversia perché tra di essi non c'è mai stato l'intenzione di dialogare. Un insegnamento possiamo tuttavia ricavarne. I filosofi, i sociologi, gli storici hanno innumerevoli cantieri in cui lavorare ma per progredire debbono imparare a dialogare senza riserve con tutti quelli che sono professionalmente interessati a comprendere i problemi della scienza, della conoscenza, e che lavorano in cantieri vicini o negli stessi. I risultati dei lavori dei dirimpettai meritano d'essere incorporati in quelli a cui riserviamo le nostre energie ed il nostro impegno ${ }^{87}$. Senza un tale dialogo i dibattiti sono improduttivi e le controversie producono solo incomprensioni ed ostilità.

\footnotetext{
85 Baigries, B.S., Les recoins de la raison: vers une sociologie cognitive de la connaissance, in: Sous la direction de Kremer-Mariotti, A., Sociologie de la science, op. cit., pp. 209-232.

86 Bourdieu, P., Les usages sociaux de la science. Pour une sociologie clinique du champ scientifique, op. cit.

87 La questione dell'utilizzazione delle ricerche storiche da parte degli scienziati, dei filosofi e dei sociologi non è stata mai discussa con attenzione benché l'argomento sia d'una importanza capitale. Per una prima informazione ved. Bourdieu, P., La cause de la science. Comment l'histoire sociale des sciences sociales peut servir le progrès de ces sciences, «Acte de la recherche en sciences sociales » $\mathrm{n}^{\circ}$ 106-107, mars 1995, pp. 3-10.
} 


\section{POSTILLA}

Questo testo fu alla base d'una serie di lezioni date nel marzo 1999 nell'Istituto Italiano per gli studi storici di Napoli, su invito del suo direttore, il professore Gennaro Sasso. Pubblicato, nel settembre dello stesso anno, nel fascicolo III del volume CXI della «Rivista storica italiana», il suddetto testo è qui riprodotto immutato con l'intento di ricordare che il dialogo auspicato resta ancora di là da venire. Finora tutto fa presumere che i contendenti non possano né vogliano dialogare, né a corta scadenza né a medio termine, dato il groviglio d'ostilità, d'inimicizie, d'incomprensioni, vista la molteplicità dei processi intenzionali, dei rifiuti d'ammettere la buona fede dei rivali, le critiche immaginate, percepite e ricevute come aggressioni ad personam.

Il caso di Bruno Latour è diventato ormai paradigmatico. Costui percepisce le osservazioni a proposito dei suoi lavori come se fossero unicamente critiche aspre, malevoli, violente, insensate alla sua persona. Perciò le ricusa tutte («At least they spelled my name right !»), rifiuta di discutere gli argomenti dei «guerrieri della scienza» perché «ils attaquent quelqu'un réputé défendre des absurdités », mentre lui da almeno un quarto di secolo non avrebbe mai difeso la tesi che la scienza è una mera costruzione sociale, non avrebbe mai scritto che tutto è discorso, che la realtà esterna non esiste, che la scienza è sprovvista di contenuti concettuali, che tutto è politico e che «tout se vaut».

Non si capisce allora perché mai tanti ricercatori si ostinino ad interpretare gli scritti di Latour con tanta malevolenza, disonestà intellettuale, perché mai questi ricercatori scrivano tante «scempiaggini». Risulta misterioso, non intelligibile, comprendere perché Latour anziché schiarire gli eventuali malintesi, indicare le distorsioni dolose, correggere le letture fallaci, si limiti a lanciare solo improperi e parli unicamente delle offese di cui è la vittima, del rifiuto ostinato della corporazione dei sociologi di riconoscere alle sue ricerche l'originalità e la novità di cui lui, invece, è assolutamente certo. Latour non fa altro che ripetere d'aver risituato la scienza nella vita reale, d'averla aperta alla cultura umanistica, d'aver valorizzato il ruolo degli uomini nell'elaborazione dei fatti scientifici e rivelato l'importanza delle scienze nella fabbricazione della storia umana. Le critiche rivoltegli non sarebbero, in più di tante altre cose, che tentativi per dileggiare e poi privare di legittimità le scienze umane e sociali.

Le accuse di relativismo sono respinte perché non pertinenti, quelle di riduzionismo e d'uso regolare d'un doppio linguaggio scartate perché ingiuste ed offensive. Relativizzando il relativismo, Latour dice di volere, al contrario, valorizzare il relazionismo e generalizzare l'uso di questo approccio nelle scienze dell'uomo e della società ${ }^{88}$. Ma lo stesso Latour ci avverte in pari tempo che «Tout est à remettre sur le métier: la France, le genre, la parité, l'identité, le service public, l'économie, l'appartenance, la communauté, l'inconscient, la laïcité, la religion et, bien sûr, les sciences dans leurs liens avec la politique». È stato lui stesso a scrivere che mercé l'importanza data ai pamphlet di Sokal «On avait voulu construire une gigantomachie entre les lumières de la raison universelle de

88 Per chiarire la controversia basti rileggere lo scritto di 1961 di Strauss, L., Le «relativisme», ripubblicato ora in «Le genre humain», 1986, n 14, pp. 191-209. 
la science et l'obscurantisme auquel menait tout droit le relativisme [...] Faux combat dont le piège fut vite éventé: le lien des sciences, des cultures, des politiques et des Nations, dès qu'on le rend un peu complexe, permet de reconstituer un tout autre histoire des sciences et, partant, un tout autre distribution entre lumières et obscurité ${ }^{89}$ Latour reputa che l'incertezza, il carattere controverso e provvisorio dei risultati delle ricerche rendono la perizia scientifica inadeguata e inadatta all'elaborazione delle scelte politiche, delle decisioni collettive. Appunto perciò sta avvenendo, senza che noi ce ne rendiamo conto, una riorganizzazione profonda della vita politica e dell'azione collettiva grazie alla quale sparirà prossimamente «la dispute surannée sur la façon de contrôler au mieux le peuple». Ormai una sana politica «exige que l'on agisse pour d'autres raisons et selon d'autres critères que la connaissance précise des causes et des conséquences, même lorsqu'il s'agit d'imbroglios des sciences et des techniques ». ${ }^{90}$

Sembra evidente che la preoccupazione maggiore di Latour sia quella d'elaborare una filosofia politica, «une politique des choses» capace di liquidare «la dispute surannée sur la façon de contrôler mieux le peuple». Ovviamente egli rifiuta di confondere la politica con i pubblici poteri e con il governo poiché «l'État est tout aussi superficiel et formaté que le marché» ${ }^{91}$. Appunto per sbarazzarsi di queste aporie l'antropologia delle scienze gli serve per riformulare un nuovo concetto d'attività politica, d'azione umana e sociale, le quali finora hanno trovato la loro giustificazione ultima nella natura, nella scienza e nella ragione.

La natura, fondamento esterno, evidenza, ritenuta un'essenza differente dalla società con le proprie leggi specifiche, i fatti opposti ai valori, la scienza all'ideologia, il soggetto all'oggetto, le opposizioni natura-cultura, razionale-irrazionale, sarebbero stati ipostatizzati dal « compromesso modernista». Quest'ultimo dissimulerebbe il fatto che trattisi, invece, d'artefatti separanti l'esterno (la natura) dal dentro (lo spirito), il basso (il sociale) dall'alto (Dio). Tale « compromesso modernista» avrebbe «verrouillé dans des problèmes séparés des questions qui ne peuvent être résolues séparement et doivent être affrontées toutes en même temps » tanto più che si tratta, in verità, dei problemi capitali di come conoscere il mondo esterno, di come la mente riesce a mantenere un legame esso, di come assicurare l'ordine sociale, di come vivere una «buona vita». Inoltre, il detto «compromesso modernista » avrebbe creato una «polizia epistemologica» incapace di prendere in conto i non-umani che pigiano da tutte le parti, che non sa affrontare le situazioni in cui gli umani ed i non-umani (effetto serra, la vacca pazza, ecc.) s'incrociano, «polizia epistemologica » assolutamente inadatta a superare la crisi permanente dell'oggettività.

89 Latour, B., et Alii, Répenser la Republique, «Le Monde», 3 febbraio 2000.

90 Latour, B., Prenons garde au principe de précaution, «Le Monde», 4 gennaio 2000

91 Cfr. Callon, M. et Latour, B., «Tu ne calculera pas!» ou comment symétriser le don et le capital, in «Revue du Mauss semestrielle», $\mathrm{n}^{\circ}$ 9, premier semestre 1997, pp.45-70, dove già si legge: «[...] l'étude anthropologique des instruments scientifiques étendue à tous les 'valorimètres'nous permettra de reformuler le débat entre libéralisme et anti-utilitarisme, d'évaluer autrement le rôle permormatif des sciences économiques et sociales, enfin de réutiliser l'anthropologie symétrique pour établir d'autres règles de conduite vis-à-vis de ce monstre aux mille bras que l'on appelle un peu vite 'marchémondial'.» (p. 45). Vedere anche Caillé, A., Brève replique à Michel Callon et Bruno Latour, Ibid., pp. 71-76. 
La divisione natura/società occulterebbe, insomma, la realtà del processo politico reale e metterebbe in non cale il fatto che «le cosmos est rassemblé dans un tout où il soit possible de vivre[...] Sa devise pourrait être 'pas de réalité sans représentation'». La realtà unisce gli umani e i non-umani, non esclude né l'esteriorità né la distinzione, prende atto che gli uomini e le cose esistono in quanto tali, non come degli a priori assoluti, ma come prodotti di dibattiti, di consensi e d'adesioni. Gli umani ed i non-umani sono esseri di diritto e non già essenze. Il relativismo/relazionismo non sarebbe niente altro che un processo nel corso del quale, mercé la mediazione di strumenti (eventi, attori indefinibili dagli input e output, ibridi, oggetti, quasi-oggetti, reti, ecc.), si stabiliscono delle relazioni tra punti di vista divergenti e disparati. Le credenze non sono né uno stato psichico né un mezzo per comprendere gli enunciati, ma solo un modo di relazione polemico prodotto dalla distinzione tra la costruzione e la realtà.

Se leggiamo i lavori di Latour come un tentativo in vista dell'elaborazione d'una filosofia politica suscettibile di risolvere molte questioni che il movimento ecologistico ha posto senza tuttavia arrivare finora a dare risposte convincenti, se ammettiamo che questa filosofia politica pretende fare ammettere il riconoscimento della natura politica dell'attività scientifica, che vuole sottrarre l'azione collettiva ad una speciosa tecnicizzazione e mettere l'accento su tutto ciò che è imponderabile, rischioso, e sulla molteplicità dei tipi d'azione, di contesti, ecc.ecc., allora molte di queste dispute diventano superflue. Però Latour non dà nessun contributo specifico e fondamentale alla sociologia delle scienze né alla sociologia della conoscenza; ha il merito d'aprire nuove piste nel territorio dell'odierna asfittica riflessione politica ${ }^{92}$.

Certo, Latour è un uomo con un forte carattere, che arriva a suscitare ammirazione o ostilità, un personaggio che s'è accorto che il dibattito scientifico è ormai spettacolo, talk-show, che le regole dei mass-media hanno rimpiazzato quelle messe a punto dalla razionalità scientifica; è un filosofo che sa utilizzare con maestria meiosi, tropi, tautologie, prolessi e tutti gli altri accorgimenti retorici per aver ragione, sedurre, e che s'è accorto che in questo mondo d'illusioni e simulacri per apparire originale è necessario profferire enormità e autopresentarsi come la vittima delle ottusità e delle malevolenze dei misoneisti. Perciò s'infiamma e tuona, sa irritare ed essere cattivo, non indugia sui mezzi da utilizzare, riesce magistralmente a nascondere d'essere autoritario, retorico, contraddittorio, vanitoso ed anche tendenzialmente ambizioso e provocatore. Si deve riconoscere tuttavia che Latour non è un ipocrita. Dice e scrive, con energia e vigore, quello che crede veramente e fortemente. I suoi scritti sono incisivi, impegnati, talvolta iconoclastici, quasi sempre ben costruiti. Questo filosofo ha scelto, definitivamente, la passione e la deplorazione contro la freddezza ed i rallegramenti. E di ciò deve dargliersene atto colla più grande delle lealtà ${ }^{93}$.

92 A questo proposito leggere le pagine, non tutte convincenti, de Caillé, A., Une politique de la nature sans politique. A propos de 'Politiques de la nature' de Bruno Latour, «Revue du Mauss semestrielle», $\mathrm{n}^{\circ}$ 17, premier semestre 2001, pp. 94-116. Altre osservazioni interessanti ma discutiibili in Vandenberghe, F., Reconfiguration et rédemption des acteurs en réseaux. Critique humaniste de la sociologie actantielle de Bruno Latour, Ibid., pp. 117-136.

93 Una testimonianza lampante, inutilmente caustica, furbescamente spiritosa, di quanto sopra scritto si trova in Latour, B., Réponse aux objections..., «Revue du Mauss semestrielle », $\mathrm{n}^{\circ} 17$, 
Un eccellente contributo al dibattito pacato è dato, invece, da Michel Dubois con un florilegio di suoi articoli riuniti in un volume, dove, purtroppo, sono stati omessi gli indici dei temi e dei nomi ${ }^{94}$. È spiacevole che libri di questa importanza siano sprovvisti degli apparati che rendono agevole la consultazione.

Il volume ritraccia la storia della progressiva affermazione del «programma forte » e del «programma duro », in questo libro denominato « costruttivismo ». Le vicende e peripezie del dibattito francese sono riferite con minuzia e precisione. Molte pagine sono riservate alla natura della spiegazione sociologica ed ai diversi modelli di spiegazione utilizzati dai sociologi della scienza. Il Dubois dimostra quanto profondo sia il fossato tra le teorizzazioni della spiegazione ed i suoi impieghi nelle ricerche empiriche, dove i principi di causalità e di simmetria non sono mai rispettati. Situa poi lo sviluppo dell'approccio «costruttivista» delle scienze in una prospettiva storica lunga e ne fa la continuazione del «movimento critico delle scienze » in auge in Francia verso la fine del secolo XIX ${ }^{\circ}$. Per quanto riguarda l'opzione relativista, Dubois elenca le molteplici rappresentazioni del senso, la scarsa attenzione prestata al controllo sperimentale, gli a priori a proposito dei modelli di riferimento comune e delle strutture integratrici ed unificatrici, le utilizzazioni letterariamente poco rispettose dei lavori antropologici. I capitoli 6 e 7 raccontano come il «programma forte » ed il « costruttivismo » hanno reagito nel corso del dibattito aperto da Sokal. Dubois indica bene i limiti del sociocostruttivismo e senza mai dire come Brown che «Latour ne nous offre que le cynisme ${ }^{95}$, pure conclude maliziosamente il suo dire così: « Les sociologues constructivistes ont fréquemment souligné les méthodes parfois peu scrupuleuses utilisées par les scientifiques pour clôre à leur bénéfice des querelles de priorité dans le cas de découvertes apparemment simultanées. Pour autant, ils n'ont que très rarement pris conscience du caractère hautement improbable de ces querelles au regard de leur propre représentation des sciences ${ }^{96}$.

Al travisamento del dibattito aperto da Sokal, alle polemiche improduttive da esso prodotte, agli sterili, inutili, enunciati da esso derivati, alle occasioni mancate di chiarire tante questioni epistemologiche importanti, è dedicato il libro curato

premier semestre 2001, pp. 137-151. Si comincia col dire «At least they spelled my name right!», si continua coll'affermare «Depuis vingt-cinq ans, j'ai essayé de rendre un peu de réalisme aux sciences sociales, en les faisant échapper au choix délétère entre symbolique d'une part, et la dure infrastructure d'autre part. J'ose avancer qu'avec la sociologie de la traduction, la sociologie est aujourd'hui un peu moins 'acosmique' qu'elle ne l'était avant nous; en tous cas, nous sommes peu nombreux à ne pas confondre l'enfilage de briques avec l'enfilage de jeux de langage.» (p. 141) Infine Latour scrive che la società è un gigantesco laboratorio la cui sola legittimità deriverebbe dalla sperimentazione permanente: «L laboratoire est devenu en effet le monde, et il nous manquent les règles de la méthode expérimentale permettant de suivre cette expérimentation collective exercée pour l'instant en dépit du bon sens. C'est justement parce qu'il nous faut des gardefous que j'ai entrepris de rédiger les règles de la méthode pour une expérience collective étendue au-delà du laboratoire.[...] Pour se convaincre que l'alternative est bien de vivre dans un laboratoire étendue à la planète où l'on expérimente sur nous tous sans nous demander notre avis, ou bien de faire basculer l'ensemble du laboratoire dans l'arène politique, il suffit de regarder le journal télévisé.» (p.146)

Dubois, M. La nouvelle sociologie des sciences, Paris, PUF, 2001.

95 Browwn, J.R., La science prosaïque de Bruno Latour, in Kremer-Mariotti, A., Sociologie de la science, op. cit., p. 168.

96 Dubois, M., op. cit., p. 256. 
dalla Kremer-Mariotti ${ }^{97}$ nel quale si leggono contributi importanti come quelli, ad esempio, di Jacqueline Feldman (Les savoirs aujourd' hui : Pour un travail d'explicitation, pp. 39-64) o della stessa Kremer Mariotti (pp.11-24 e pp. 279-301) che opportunamente ricorda che le scienze umane si trovano oggi in una «impasse épistémologique», che le polemiche attuali sono gli indicatori d'una crisi difficile da cui potremmo uscire se avremo il coraggio d'affrontare i «véritables problèmes d'objet et de méthode» e se finalmente accetteremo il saggio principio «qu'affirmer n'est pas argumenter et, de plus, qu'argumenter scientifiquement implique de soutenir une référence objectivement constatable et, dans le meilleur des cas, quantifiable.» Il relativismo cognitivo o epistemico, « dissipe toute détermination précise et exacte en une vague et indéfinie réflexion, sans compter qu'il peut entraîner les pires conséquences morales et politiques, et servir, en tant que relativisme socioculturel, 'de paravent et d'excuse idéologique aux pires horreurs historiques ou politiques'.»

Se il dibattito aperto da Sokal non fosse stato impaludato da personalismi narcisistici e da interessi corporativi, la questione della scienza e della parascienza, non sarebbe più l' «objet volant non identifié ${ }^{98}$ e forse saremmo capaci di tematizzare talune problematiche essenziali delle scienze sociali, le quali purtroppo, e lo diciamo colle stesse parole della Stengers, «dans la mesure où elles n'ont pas affaire à un terrain capable de maintenir son identité quels que soient les soupçons de l'enquêteur ou les inventions du juge (l'humain est influençable, son identité intégre activement le traitement qu'il subit), ne puissent faire l'économie de cette thématisation. Il serait historiquement très amusant - si on oserait l'espérer - que soit à propos des possibilités de passage de la (non)histoire à l'histoire des phénomènes parapsys que cette thématisation puisse s'inventer $»^{99}$.

Tematizzare ma in che modo? Come mettere insieme delle cose tante diverse? Il caso di Isaac Newton dovrebbe spingerci alla prudenza. William Stukeley e Sir David Brewster hanno trasformato lo scienziato inglese in un genio incomparabile. Nel 1942 John Maynard Keynes ha distrutto questo mito d'un Newton grandioso speculatore e sperimentatore razionalista rigorista. «Newton non fu il primo scienziato dell'età della ragione. Piuttosto fu l'ultimo dei maghi, l'ultimo dei Babilonesi e dei Sumeri.» Alchimista, occultista, eretico antitrinitario, omosessuale (è documentata la sua relazione tempestosa col matematico svizzero Nicolas Fatio de Duillier), è notorio che Newton apprezzava solo i filosofi della natura che accettavano le sue ipotesi di lavoro e che non si permettevano di contestare la sua priorità teorica. La disputa con Leibniz sulla paternità del calcolo infinitesimale prova che era ossessionato dall'idea che «nella medesima epoca non vi potesse essere più di un glorioso interprete del cosmo ». Ma come dimenticare che in punto di morte confidava al nipote Benjamin Smith: «Non so cosa posso sembrare al mondo; ma a me stesso sembra di essere stato come un ragazzo che gioca sulla riva del mare e si diverte a trovare di quando in quando un ciottolo più liscio

97 Sous la direction de Kremer-Mariotti, A., Éthique et épistémologie autour du livre «Impostures intellectuelles» de Sokal et Bricmont, Paris, L'Harmattan, 2001.

98 Lagrange, P., La sociologie à l'épreuve des parasciences, «Ethnologie française», 23, 1993, n 3, pp. 311-312.

99 Stenger, I., Conditions pour une histoire, Ibid., pp. 459-463. 
o una conchiglia più bella, mentre il grande oceano della verità si stende ignoto tutt'intorno.» (David H. Clark and Stephen P.H. Clark, Newton's Tiranny, New York, Freeman, 2001.)

Qual è l'importanza di tutto ciò rispetto al calcolo delle flussioni, alle tre leggi della termodinamica, alla teoria della gravitazione? Come dimenticare che la fisica meccanica di Newton resta alla base della scienza, eccezion fatta per due casi estremi (le velocità prossime della luce ed il mondo subatomico)? Possiamo dire e dimostrare che la sua alchimia ed il suo occultismo, insomma le parascienze costituiscano delle protoscienze? Ma è accettabile, sul piano della logica, la sostituzione del criterio di verità con quello di pertinenza, ridurre il senso (attribuito da un attore, ad un dato momento, a qualcosa d'insolito e di misterioso) a credenza?

Certo, i limiti della razionalità classica sono stati, oggi, in parte cancellati dalle interpretazioni che hanno esteso all'infinito il campo della conoscenza. Se vogliamo evitare di cadere nei sofismi bisogna continuare ad usare correttamente i due classici argomenti standard (il ragionamento sillogistico e argomenti che s'appoggiano su esempi verificabili) e non dimenticare che comunicare implica un certo modo di conoscere la realtà, che conoscenza e funzione sono intimamente collegate, che le strutture determinanti i nostri modi di conoscere rendono possibile anche il nostro fare, le nostre pratiche, che le scienze umane hanno per oggetto non una realtà materiale ma soprattutto i modi di conoscerla e di modificarla coll'azione pratica.

Tutti questi malintesi sono ritornati alla luce in occasione della «soutenance de thèse », nell'Università René Descartes-Paris-V, sull'astrologia, della signora Elisabeth Teissier. Baudelot e Establet hanno scritto che il direttore della tesi, Michel Maffesoli, «s'est toujours singularisé par ce refus [de l'objectivation] en privilégiant le culte du vécu, l'interprétation gratuite et l'analyse spontanée». Dando il proprio avallo accademico, egli accredita la tesi assurda d'una certa equivalenza tra l'astrologia e la sociologia ${ }^{100}$.

Bisogna osservare che siamo, con questa tesi, molto lontani dai tempi (1971) in cui Edgar Morin realizzò l'inchiesta Le retour des astrologues. Diagnostic sociologique, con Fischler, C., Defrance, Ph., Petrossian, L., ora ristaampata in La croyance astrologique moderne. Diagnostic sociologique (Lausanne, Ed. L'Âge d'Homme, 1981, IIa edizione).

Bourdin, da parte sua, ha scritto che il dibattito mette in evidenza la crisi da cui sono afflitte le scienze sociali. «Laisser préparer une thèse qui sort des sentiers habituels au monde académique n'est pas un crime. Il faut juste de la rigueur et de l'éthique. En effet, aucune science ne peut progresser sans prendre des risques et sans accepter à certains moments l'émergence de discours totalement hétérodoxes. Il s'agit alors d'instaurer un débat -souvent violent- entre les chercheurs. L'objectif de l'innovateur et de ceux qui le soutiennent est de parvenir à convaincre ses collègues avec des arguments qui soient recevables par eux.» ${ }^{101}$. Anche in quest'occasione la dialettica dialogica non ha funzionato, non è stata applicata. Maffesoli si limita a pronunciare l'elogio della conoscenza ordinaria, a proclamare che «Une raison ouverte à l'imaginaire, au ludique, à l'onirique social est

100 Baudelot, C. et Establet, R., La sociologie sous une mauvaise étoile, «Le Monde », 18 aprile 2001.
101 Bourdin, A., La sociologie, l'antithèse de Teissier, «Libération », 19 aprile 2001. 
autrement plus riche en ce qu'elle sait intégrer, homéopathiquement, cette ombre qui aussi nous constitue.» ${ }^{102}$

Copans, dal canto suo, allarga il dibattito quando scrive:«C'est la sociologie (et de plus en plus l'ethnologie) qui a 'astrologisé' ses compétences pour un plat de lentille! Depuis vingt ans, ces deux sciences sociales se sont vendues aux pouvoirs, aux administrations et aux médias qui nous gouvernent pour expliquer (et, sous-éntendu, prévoir) le présent et le futur proche.» ${ }^{103}$ Secondo Pirioux le discussioni sollevate dalla tesi della signora Teissier ripropongono un vecchio dibattito, mai risolto, tra scientisti metodologici e saggisti estetizzanti, ed in pari tempo sollevano una questione, finora sapientemente dissimulata, quella del «l'enjeu du renouvellement de questions plus corporatistes concernant l'organisatiom de la sociologie comme profession, au contrôle de l'accès au métier.»104

Da quest'ultimo punto di vista, le reazioni sono state vivacissime, non sul piano epistemologico ma su quello dei criteri utilizzabili per riconoscere la professionalità di coloro che vogliono praticare la sociologia come mestiere ${ }^{105}$.

Alain Touraine ha chiuso saggiamente, almeno per il momento, il dibattito, con considerazioni che non si possono non condividere pienamente: «J'ai moimême, comme la plupart des sociologues, une réaction de vif rejet à l'égard de l'irrationalisme et des efforts faits pour relativiser la science et la mettre sur le même plan que n'importe quel type de connaissance.[...] Les sciences sociales ne sont pas des non-sciences de la nature. [...] Nous ne savons plus découvrir l'unité de l'univers et nous devons de toute urgence revenir à des modes de connaissance capable de nous découvrir, à travers notre expérience vécue, sensuelle et émotive autant qu'intellectuelle, les mondes que nous a caché la science. Il faut aller audelà de la raison et de ses calculs; il faut comprendre - mais en ne donnant pas à ce mot le sens que lui a donné Weber -, en participant, en appartenant. Et nous allons heureusement sortir de ce scientisme et rencontrer que c'est l'être tout entier qui doit produire la connaissance, et pas seulement l'esprit. On a reproché sans raison à Mme Teissier de consacrer sa thèse à une fausse science; en fait, elle ne l'a consacrée qu'à elle-même.» ${ }^{106}$

Purtroppo è quello che succede di più in più sovente nei dibattiti epistemologici e di sociologia della conoscenza, e più particolarmente in sociologia ed antropologia delle scienze. Speriamo che questo vezzo distruttore dei dibattiti sereni scomparirà presto e che gli studiosi riprenderanno a dialogare senza riserve mentali, senza asprezze di toni, animati dalla fiducia che i colleghi che la pensano diversamente non sono necessariamente dei nemici aggressivi e cattivi.

Il che è una necessità imperiosa in modo particolare in quest'epoca in cui è dominante la credenza che la scienza è la base stessa dei cicli economici, che la

102 Maffesoli, M., Eloge de la connaissance ordinaire, «Le Monde», 24 aprile 2001.

103 Copans, J., La sociologie, astrologie des sciences sociales, «Le Monde», 2 maggio 2001.

104 Pirioux, O., Banalité d'Elizabeth Teissier, «Le Monde», 2 maggio 2001.

105 Morin, H., La thèse d'Elizabeth Teissier ravive la fracture au sein de la sociologie, «Le Monde», 5 maggio 2001, ma anche Moliterni, R., Di che segno è la Sorbona ?, «La Stampa», 8 maggio 2001. Ritorna, infine, sull'argomento Morin, H., La sociologie miroir de la thèse d'Elizabeth Teissier, «Le Monde», 15 maggio 2001.

106 Touraine, A., De quoi Elizabeth Teissier est-elle coupable ?, Le Monde », 22 maggio 2001. 
separazione tra la ricerca fondamentale e la ricerca applicata è nociva per lo sviluppo, per gli investimenti e per i redditi.

Il processo di fusione della scienza coll'industria sta dando a quest'ultima il diritto di valutare la ricerca come se si trattasse d'un investimento speculativo qualsiasi. Appunto perciò la biologia molecolare e le biotecnologie sono diventate oggi le discipline le più massicciamente finanziate, le più favorite nei programmi universitari di sviluppo, come ieri lo sono state, senza discernimento e coi magri risultati che oggi conosciamo, l'energia nucleare e l'intelligenza artificiale.

I sociologi delle scienze saranno capaci d'affrontare il problema costruttivismo/naturalismo senza pregiudizi, d'oggettivare queste pretese ideologiche specifiche ai nostri tempi e distinguerle dai cambiamenti di contenuti nei sistemi cognitivi se e soltanto se ritorneranno a praticare la virtù del dialogo, lo scetticismo organizzato, a non confondere la logica della dimostrazione con quella dell'argomentazione, i linguaggi formali coi linguaggi naturali? Un segno positivo in questa direzione giunge da Dominique Pestre quando propone d'abbandonare le opposizioni costruttivismo/naturalismo, realismo/essenzialismo, di ripensare la problematica dell'attore sociale, di meglio analizzare le attività di conoscenza e di classificazione di «tout ce qui contribue à la fixation des significations (la matérialité des choses par exemple)», di mostrare un'attenzione maggiore alla «dimension réflexive de l'activité humaine (et de la nôtre en particulier).» È il prezzo che bisogna pagare, per dirla col Pestre, affinché «Renaissent alors d'autres préoccupations intellectuelles -sur ce que nous faisons, sur les présupposés qui encadrent nos questions et les orientent d'entrée de jeu; alors renaît l'inquiétude salutaire car déstabilisatrice de nos certitudes.» ${ }^{107}$ Altre voci fanno eco e ben augurare dei prossimi chiarimenti, indispensabili al rinnovamento delle ricerche ed al ritorno nella comunità scientifica d'un clima meno tempestoso, dei presupposti ontologici, epistemologici e metodologici della discussione sulle scienze e sul ruolo delle discipline che studiano le scienze ${ }^{108}$.

Institut d' anthropologie et de sociologie

Université de Lausanne

107 Pestre, D., Études sociales des sciences, politique et retour sur soi. Éléments pour sortir d'un débat convenu, in «Revue du Mauss semestrielle», $\mathrm{n}^{\circ}$ 17, premier semestre 2001, pp. 180-196.

108

Cito, come esempi di questo rinnovo, due lavori recenti: Dubois, M., Le relativisme cognitif comme «moulin à vent»? Constructivisme et relativisme en sociologie des sciences, in: KremerMariotti, A., Éthique et épistémologie autour du livre «Impostures intellectuelles» de Sokal et Bricmont, Paris, L'Harmattan, 2001, pp. 109-135; Gingras, A., Pourquoi le «programme fort» est-il mal compris?, "Cahiers internationaux de sociologie», CIX, Juillet-Décembre 2000, pp. 235-255. 